\title{
Infinitesimal invariants for cycles modulo algebraic equivalence and 1-cycles on Jacobians
}

\author{
Claire Voisin
}

\begin{abstract}
We construct an infinitesimal invariant for cycles in a family with cohomology class in the total space lying in a given level of the Leray filtration. This infinitesimal invariant detects cycles modulo algebraic equivalence in the fibers. We apply this construction to the Ikeda family, which gives optimal results for the Beauville decomposition of the 1-cycle of a very general plane curve in its Jacobian.
\end{abstract}

\section{Introduction}

This paper is devoted first of all to the construction of infinitesimal invariants for (families of) cycles modulo algebraic equivalence, similar to the infinitesimal invariants used in [Voi94b] for the study of families of cycles modulo rational equivalence, and secondly to a geometric application of these infinitesimal invariants to the study of the length of the Beauville decomposition of the 1-cycle of a curve in its Jacobian.

Infinitesimal invariants appear in the following situation: Let $\pi: \mathcal{Y} \rightarrow B$ be a smooth projective morphism, and let $\mathcal{Z}$ be a codimension $n$ cycle in $\mathcal{Y}$. We assume that the cohomology class $[\mathcal{Z}] \in H^{2 n}(\mathcal{Y}, \mathbb{Q})$ belongs to the $s$-th level of the Leray filtration on $H^{2 n}(\mathcal{Y}, \mathbb{Q})$. Then we produce for any point $b \in B$ an infinitesimal invariant $\delta[\mathcal{Z}]_{b, a l g}$ which depends only on the first order neighborhood $\mathcal{Z}_{b, 2}:=\mathcal{Z}_{\mid \mathcal{Y}_{b, 2}}$ of the restricted cycle $\mathcal{Z}_{b}:=\mathcal{Z}_{\mid \mathcal{Y}_{b}}$ of the fiber $\mathcal{Y}_{b}$ (hence the name "infinitesimal invariant"). More precisely, $\mathcal{Z}$ has a Dolbeault cohomology class $[\mathcal{Z}]^{n, n} \in H^{n}\left(\mathcal{Y}, \Omega_{\mathcal{Y}}^{n}\right)$ and this infinitesimal invariant will depend only on the restricted class

$$
[\mathcal{Z}]_{\mid \mathcal{Y}_{b}}^{n, n} \in H^{n}\left(\mathcal{Y}_{b}, \Omega_{\mathcal{Y} \mid \mathcal{Y}_{b}}^{n}\right) \text {. }
$$

This infinitesimal invariant has the property that it allows us to decide whether the restricted cycle $\mathcal{Z}_{b}$ is algebraically equivalent to 0 at the very general point $b \in B$. More precisely, we will prove the following result.

THEOREM 1.1. In the situation above, assuming that $s \geqslant 2$ and $[\mathcal{Z}] \in L^{s} H^{2 n}(\mathcal{Y}, \mathbb{Q})$, there is an infinitesimal invariant $\delta[\mathcal{Z}]_{\text {alg }}$ which is a section of the sheaf

$$
\frac{\operatorname{Ker} \bar{\nabla}_{s, n-s, n}}{\Omega_{B} \wedge \operatorname{Ker} \bar{\nabla}_{s-1, n-s, n}},
$$

\footnotetext{
Received 15 April 2013, accepted in final form 9 September 2013. 2010 Mathematics Subject Classification 14C25, 14D07, $14 \mathrm{~K} 99$ Keywords: Algebraic cycles, infinitesimal invariants, Jacobians This journal is (c) Foundation Compositio Mathematica 2014. This article is distributed with Open Access under the terms of the Creative Commons Attribution Non-Commercial License, which permits non-commercial reuse, distribution, and reproduction in any medium, provided that the original work is properly cited. For commercial re-use, please contact the Foundation Compositio Mathematica.
} 


\section{INFINITESIMAL INVARIANTS AND 1-CYCLES IN JACOBIANS}

with restriction

$$
\delta[\mathcal{Z}]_{b, a l g} \in \frac{\operatorname{Ker} \bar{\nabla}_{s, n-s, n, b}}{\Omega_{B, b} \wedge \operatorname{Ker} \bar{\nabla}_{s-1, n-s, n, b}},
$$

which is 0 at a general point $b \in B$ if for general $b \in B, \mathcal{Z}_{b}$ is algebraically equivalent to 0 up to torsion in the fiber $\mathcal{Y}_{b}$.

The maps

$$
\bar{\nabla}_{s, n-s, n}: \Omega_{B}^{s} \otimes \mathcal{H}^{n-s, n} \rightarrow \Omega_{B}^{s+1} \otimes \mathcal{H}^{n-s-1, n+1}
$$

are induced by the infinitesimal variation of Hodge structure on the cohomology of the fibers of $\pi$; they are defined using the Gauss-Manin connection and the Hodge filtration on Hodge bundles associated to the family $\mathcal{Y} \rightarrow B$ (cf. [Gri83], [Voi03, 5.1.2]).

Since the set of points $b \in B$ such that a multiple of the restricted cycle $\mathcal{Z}_{b}$ is algebraically equivalent to 0 is a countable union of closed algebraic subsets of $B$, we can rephrase Theorem 1.1 as follows.

Corollary 1.2. There is a Zariski open set $U \subset B$ such that if $\delta[\mathcal{Z}]_{b, a l g} \neq 0$ for some $b \in U$, then $[\mathcal{Z}]_{b^{\prime}}$ is not algebraically equivalent to 0 up to torsion for very general $b^{\prime} \in B$.

More precisely, we can take for $U$ the Zariski open subset of $B$ consisting of points where the sheaf $\operatorname{Ker} \bar{\nabla}_{s, n-s, n} /\left(\Omega_{B, b} \wedge \operatorname{Ker} \bar{\nabla}_{s-1, n-s, n}\right)$ is locally free; the nonvanishing of $\delta[\mathcal{Z}]_{b, a l g}$ for some $b \in U$ then guarantees the nonvanishing of $\delta[\mathcal{Z}]_{b, a l g}$ for general $b \in B$.

The main difference between the present construction and the one in [Voi94b] lies in the fact that the infinitesimal invariant introduced here detects cycles modulo algebraic equivalence, while in our previous work only cycles modulo rational equivalence (a much bigger group) could be detected. There is a serious difficulty here, since Lemma 2.2, which says that up to shrinking the base $B$, the cycle $\mathcal{Z}$ as above is cohomologous to 0 modulo torsion on the total space $\mathcal{Y}$ if the restricted cycles $\mathcal{Z}_{t}$ are rationally equivalent to 0 , obviously does not hold when replacing rational equivalence by algebraic equivalence.

Our infinitesimal invariants are not present, at least not explicitly, in the work of Nori [Nor93] and Fakhruddin [Fak96], but they are in fact hidden in the infinitesimal variations of Hodge structures arguments used in their computations of the Leray filtration (we refer to [Voi94a], [Voi03] for the relation between infinitesimal invariants and Leray filtration). The work of Ikeda [Ike03] involves similar infinitesimal invariants, which, however, lie in a bigger cohomology group and allow him to detect the nontriviality of cycles modulo a relation which is much finer than algebraic equivalence. His computations have been extended by Pirola and Rizzi in [PR07].

Our main application concerns the length of the Beauville decomposition of the canonical 1-cycle of a generic Jacobian. To avoid heavy notation, we denote by $\mathrm{CH}^{i}(Y)$ the Chow groups of a variety $Y$ with rational coefficients. Recall that Beauville defined in [Bea86] a canonical decomposition on the Chow groups $\mathrm{CH}^{i}(A)$ of an abelian variety, as a direct sum of eigenspaces for the actions of the homotheties:

$$
\mathrm{CH}^{i}(A)=\oplus_{s} \mathrm{CH}^{i}(A)_{s},
$$

where

$$
z \in \mathrm{CH}^{i}(A)_{s} \Leftrightarrow \mu_{m}^{*} z=m^{2 i-s} z, \forall m \in \mathbb{Z} .
$$

Here for $m \in \mathbb{Z}$ we denote by $\mu_{m}$ the multiplication by $m$ on $A$. This decomposition works as well for cycles modulo algebraic equivalence. It should be understood as a canonical splitting 


\section{Claire Voisin}

of the conjectural Bloch-Beilinson filtration, which is supposed to be a decreasing filtration $F^{l} \mathrm{CH}^{i}(A)$ on $\mathrm{CH}^{i}(A)$, whose graded pieces $G r_{F}^{l} \mathrm{CH}^{i}(A)$ are governed by the Hodge structures on the cohomology groups $H^{2 i-l}(A, \mathbb{Q})$, and which finishes, more precisely $F^{i+1} \mathrm{CH}^{i}(A)=0$.

It is not known, however, whether the filtration defined by

$$
F^{l} \mathrm{CH}^{i}(A)=\oplus_{s \geqslant l} \mathrm{CH}^{i}(A)_{s}
$$

satisfies the axioms of a Bloch-Beilinson filtration, and in particular whether

$$
F^{1} \mathrm{CH}^{i}(A)=\mathrm{CH}^{i}(A)_{h o m} .
$$

Here the inclusion $F^{1} \mathrm{CH}^{i}(A) \subset \mathrm{CH}^{i}(A)_{h o m}$ is easy to see: for $s>0, \mathrm{CH}^{i}(A)_{s}$ is made of cycles homologous to 0 , because $\mu_{n}^{*}$ acts as multiplication by $n^{2 i}$ on $H^{2 i}(A, \mathbb{Q})$, but the reverse inclusion, which is equivalent to saying that $\mathrm{CH}^{i}(A)_{s}=0$ for $s<0$ and the cycle class map is injective on $\mathrm{CH}^{i}(A)_{0}$, is unknown.

In this paper, we consider the induced decomposition on the groups $\mathrm{CH}^{i}(A) /$ alg of cycles with rational coefficients modulo algebraic equivalence. As mentioned above, for $s>0, \mathrm{CH}^{i}(A)_{s} \subset$ $\mathrm{CH}^{i}(A)_{h o m}$, so that the $s$-th piece $\mathrm{CH}^{i}(A)_{s} /$ alg is in fact contained in the Griffiths group

$$
\mathrm{CH}^{i}(A)_{h o m} / \text { alg }=\operatorname{Griff}^{i}(A) \otimes \mathbb{Q} .
$$

We will avoid using the notation $\operatorname{Griff}^{i}(A)_{s}$ since it is used by Ikeda with a different meaning (see below). In the case where $A=J C$ is the Jacobian of a curve of genus $g$, there is a natural 1-cycle (codimension $g-1$ cycle) $Z$ on $J C$ defined as the image of $C$ under the Abel map, with respect to any chosen point or 0 -cycle of degree 1 on $C$. Being defined up to translation, it is well-defined modulo algebraic equivalence. We consider the Beauville decomposition

$$
Z=\sum_{s} Z_{s}
$$

of this cycle, with $Z_{s} \in \mathrm{CH}^{g-1}(J C)_{s} / a l g$. The study of the components $Z_{s}$ has a long history. The first result is the one by Ceresa [Cer83], saying that $Z_{1}$ is non zero in $\mathrm{CH}^{g-1}(J C)_{1} /$ alg for $g \geqslant 3$ and $C$ very general in modulus. Ceresa uses the Griffiths criterion [Gri69] that says that if a cycle is algebraically equivalent to 0, then its Abel-Jacobi invariant belongs to the maximal abelian subvariety of the intermediate Jacobian, together with a degeneration argument for the study of this Abel-Jacobi invariant. Using Griffiths's infinitesimal invariant [Gri83], an infinitesimal argument can be applied as well (cf. [CP95]) to prove the nontriviality of the primitive (or transcendental) part of the normal function given by the Abel-Jacobi image of the cycle $Z_{1}$.

In the opposite direction, there is the following vanishing result due to Colombo and Van Geemen [CvG93].

Theorem 1.3. Assume that a smooth curve $C$ has a $g_{d}^{1}$, that is, a morphism of degree $d$ to $\mathbb{P}^{1}$. Then

$$
Z_{s}=0 \text { in } \mathrm{CH}^{g-1}(J C)_{s} / a l g, \forall s \geqslant d-1 .
$$

Further results concerning the so-called "tautological ring" introduced by Beauville in [Bea04] and generated under Pontryagin product by the $Z_{s}$ have been obtained by Herbaut [Her07], Van der Geer-Kouvidakis [vdGK07], Polishchuk and Moonen [Pol07], [Moo09]. The results of Herbaut (reproved by Van der Geer-Kouvidakis) generalize the Colombo-Van Geemen result to the case where the curve admits a $g_{d}^{r}, r \geqslant 2$. This, however, does not give more vanishing for the individual components $Z_{s}$, but rather polynomial relations between them in the tautological ring (here the ring structure considered is given by the Pontryagin product of cycles). The results by Moonen 


\section{INFINITESIMAL INVARIANTS AND 1-CYCLES IN JACOBIANS}

and Polishchuk are devoted to relations in the tautological ring of a general curve, and the paper [Bea04] by Beauville describes a set of generators for this ring.

In the paper [CvG93], Colombo and Van Geemen asked for the optimality of their result, for a very general curve. (As noticed by Ben Moonen, it is not expected that their result is optimal for any curve, because for curves defined over a number field, the Beilinson conjecture [Bei87] predicts that

$$
Z_{s}=0, \forall s \geqslant 2,
$$

while the general curve defined over a number field and of genus $\geqslant 5$ has gonality $\geqslant 4$.)

Concretely, the gonality gon $(C)$ of a general curve $C$ of genus $g$, that is, the minimal degree of a $g_{d}^{1}$ on $C$, is computed using Brill-Noether theory (cf. [ACGH85]), which gives

$$
g=2 k+1 \text { or } g=2 k+2 \Rightarrow \operatorname{gon}(C)=k+2 \text { for generic } C .
$$

Thus the question asked by Colombo and Van Geemen can be stated in the following form.

Conjecture 1.4. Let $C$ be a very general curve of genus $g \geqslant 2 k+1$. Then

$$
Z_{k} \neq 0 \text { in } \mathrm{CH}^{g-1}(J C)_{k} / \text { alg. }
$$

Note that by a degeneration argument as in Ceresa's paper [Cer83], we can reduce to the case where $g=2 k+1$. Some results have been obtained in this direction. First of all, Fakhruddin [Fak96] shows the nonvanishing of $Z_{2}$ for $C$ a general curve of genus $\geqslant 11$ (the expected bound being $g \geqslant 5$ ). In a different direction, Ikeda obtains a nonvanishing result for an analogue of the cycles $Z_{s}, s \leqslant d-2$ when the curve is a very general plane curve of degree $d$ (thus of gonality $d-1)$. Colombo-Van Geemen's Theorem 1.3 says that for such a curve, the cycle $Z_{d-2}$ is zero in $\mathrm{CH}^{g-1}(J C)_{d-2} / \mathrm{alg}$. This last result is also possibly optimal since by Herbaut's above mentioned results in [Her07], we know that for plane curves, there are extra polynomial relations between the various $Z_{k}$, but a priori no further vanishing of a given component $Z_{k}$ is expected except for the vanishing of $Z_{d-2}$ given by Theorem 1.3. This leads to the following conjecture.

Conjecture 1.5. Let $C$ be a very general plane curve of degree $d$ (so $g=g(C)$ is given by $2 g-2=d(d-3))$. Then the cycle $Z_{d-3}$ is nonzero in $\mathrm{CH}^{g-1}(J C)_{d-3} /$ alg .

Ikeda addresses this conjecture but not for cycles modulo algebraic equivalence. The quotient of the group of cycles considered by Ikeda is an a priori much bigger group than the group of cycles modulo algebraic equivalence. These groups called higher Griffiths groups were defined by Shuji Saito [Sai02] and involve his construction of a Bloch-Beilinson filtration $F_{S}^{i} \mathrm{CH}^{k}(Y)$ [Sai96]. The higher Griffiths groups of Saito are then defined as the quotient

$$
\frac{F_{S}^{i} \mathrm{CH}^{k}(Y)}{F_{S}^{i+1} \mathrm{CH}^{k}(Y)+<\Gamma_{*} F_{S}^{i} \mathrm{CH}_{0}(Z)>},
$$

where $\Gamma$ runs over the set of correspondences of codimension $k$ between any smooth projective variety $Z$ and $Y$. Of course when $i=1$, the subgroup $<\Gamma_{*} F_{S}^{1} \mathrm{CH}_{0}(Z)>$ consists exactly of cycles algebraically equivalent to 0 , but for $i \geqslant 1$, the group $<\Gamma_{*} F_{S}^{i} \mathrm{CH}_{0}(Z)>\subset F_{S}^{i} \mathrm{CH}^{k}(Y)$ may be very small, and different from $F_{S}^{i} \mathrm{CH}^{k}(Y) \cap \mathrm{CH}^{k}(Y)_{\text {alg }}$. We will make a comparison between the Ikeda infinitesimal invariants and ours in Section 4, and we will see that, due to the vanishing of canonical syzygies for generic curves [Voi05], for the case of a general curve of genus $2 k+1$, his infinitesimal invariants for the cycle $Z_{s}, s \leqslant k$, highly depend on the choice of embedding of $C$ into its Jacobian (this embedding, hence also the cycles $Z_{s}$, is defined up to translation). 


\section{Claire Voisin}

Our main application of the infinitesimal invariant constructed in Section 2 is the proof of Conjecture 1.5.

Theorem 1.6. Let $C$ be a very general plane curve of degree $d$. Then

$$
Z_{d-3} \neq 0 \text { in } \mathrm{CH}^{g-1}(J C)_{d-3} / \text { alg } .
$$

Theorem 1.6 implies, in particular, the following result, which is certainly not optimal in view of Conjecture 1.4, but partially answers the question asked in [Bea04].

Corollary 1.7. Let $C$ be a very general curve of genus $g>\frac{k(k+3)}{2}$. Then the cycle $Z$ satisfies $Z_{k} \neq 0$ in $\mathrm{CH}^{g-1}(J C)_{k} /$ alg.

Proof. Indeed, we first reduce by degeneration the problem to the case where $g=\frac{1}{2} k(k+3)+1$; then we specialize $C$ to a very general plane curve of degree $k+3$ and apply Theorem 1.6. Since the specialized cycle $Z_{k}$ is not algebraically equivalent to 0 , the same is true at the very general point.

For $k=2$, the above corollary says that the very general curve of genus $g>5$ has $Z_{2} \neq 0$ in $\mathrm{CH}^{g-1}(J C)_{2} /$ alg. The bound expected from Colombo and Van Geemen's Conjecture 1.4 is $g \geqslant 5$, and as already mentioned, Fakhruddin [Fak96] proved the result in the range $g \geqslant 11$.

In order to prove Theorem 1.6, we compute the infinitesimal invariant of Theorem 1.1 on the Ikeda family of plane curves of degree $d$ (cf. [Ike03]). This family has a very special variation of Hodge structure, which makes the explicit computation quite easy.

\section{Infinitesimal invariants for cycles modulo algebraic equivalence}

\subsection{Infinitesimal invariants for cycles modulo rational equivalence}

In this subsection, we recall for the convenience of the reader the construction of infinitesimal invariants for families of cycles modulo rational equivalence. We will explain in $\S 2.2$ the modifications needed to get infinitesimal invariants for families of cycles modulo algebraic equivalence.

Let $\pi: \mathcal{Y} \rightarrow B$ be a smooth projective morphism, where $B$ is smooth quasi-projective, and let $\mathcal{Z} \subset \mathcal{Y}$ be a cycle of codimension $n$. Following Nori [Nor93], consider the cycle class

$$
[\mathcal{Z}]^{n, n} \in H^{n}\left(\mathcal{Y}, \Omega_{\mathcal{Y}}^{n}\right)
$$

with the following restriction at $t \in B$ :

$$
[\mathcal{Z}]_{t}^{n, n} \in H^{n}\left(\mathcal{Y}_{t}, \Omega_{\mathcal{Y} \mid \mathcal{Y}_{t}}^{n}\right)
$$

We have the following lemma, which will be useful later on. It is not completely obvious because we are working not on a smooth projective variety, but only on a quasiprojective one.

Lemma 2.1. The class $[\mathcal{Z}]^{n, n}$ is determined by the Betti cohomology class $[\mathcal{Z}] \in H^{2 n}(\mathcal{Y}, \mathbb{Q})$.

Proof. The class $[\mathcal{Z}]$ belongs to the pure weight $2 n$ part $W_{\text {pure }} H^{2 n}(\mathcal{Y}, \mathbb{Q})$ of the Deligne mixed Hodge structure on $H^{2 n}(\mathcal{Y}, \mathbb{Q})$, which is defined as the image of the restriction map

$$
H^{2 n}(\overline{\mathcal{Y}}, \mathbb{Q}) \rightarrow H^{2 n}(\mathcal{Y}, \mathbb{Q})
$$

for any smooth projective completion $\overline{\mathcal{Y}}$ of $\mathcal{Y}$. It is a Hodge class in $W_{\text {pure }} H^{2 n}(\mathcal{Y}, \mathbb{Q})$, which means that it comes from a Hodge class $\alpha$ on $\overline{\mathcal{Y}}$. This Hodge class can now be seen via the Hodge decomposition as a class $\alpha^{n, n} \in H^{n}\left(\overline{\mathcal{Y}}, \Omega_{\overline{\mathcal{Y}}}^{n}\right)$, which restricts to a class in $H^{n}\left(\mathcal{Y}, \Omega_{\mathcal{Y}}^{n}\right)$. If we change 


\section{INFINITESIMAL INVARIANTS AND 1-CYCLES IN JACOBIANS}

$\alpha$ to $\alpha^{\prime}$, the difference $\alpha-\alpha^{\prime}$ is a Hodge class in $\operatorname{Ker}\left(H^{2 n}(\overline{\mathcal{Y}}, \mathbb{Q}) \rightarrow H^{2 n}(\mathcal{Y}, \mathbb{Q})\right)$. It is known $(\mathrm{cf}$. [Voi14, Proof of Lemma 2.22] or [PS08, Corollary 7.3]) that if a Hodge class $\beta$ of degree $2 n$ on a smooth projective compactification $\overline{\mathcal{Y}}$ of $\mathcal{Y}$ vanishes on $\mathcal{Y}$, then there exists a Hodge class $\beta^{\prime}$ of degree $2 n-2$ on a desingularization $\bar{D}$ of $D:=\overline{\mathcal{Y}} \backslash \mathcal{Y}$ such that $j_{*} \beta^{\prime}=\beta$, where $j: \bar{D} \rightarrow \overline{\mathcal{Y}}$ is the natural morphism. It follows that $j_{*} \beta^{\prime n-1, n-1}=\beta^{n, n}$, with $\beta^{\prime n-1, n-1} \in H^{n-1}\left(\bar{D}, \Omega_{\bar{D}}^{n-1}\right)$ and thus $\beta^{n, n}$ vanishes in $H^{n}\left(\mathcal{Y}, \Omega_{\mathcal{Y}}^{n}\right)$. Applying this to $\beta=\alpha-\alpha^{\prime}$, we conclude that $\alpha^{n, n}=\alpha^{\prime n, n}$ in $H^{n}\left(\mathcal{Y}, \Omega_{\mathcal{Y}}^{n}\right)$.

The class $[\mathcal{Z}]^{n, n}$ was again considered and studied in [Voi94b], in the case of a family of 0 -cycles in a family of surfaces. Its usefulness for the study of the algebraic cycle $\mathcal{Z}$ comes from the following easy fact (see [Voi03, Theorem 10.19]).

Lemma 2.2. If for any $t \in B$, the restricted cycle $\mathcal{Z}_{t}:=\mathcal{Z}_{\mid \mathcal{Y}_{t}}$ is rationally equivalent to 0 , then there is a dense Zariski open set $U \subset B$ such that $\mathcal{Z}_{U}$ is of torsion in $C H^{n}\left(\mathcal{Y}_{U}\right)$, hence in particular

$$
[\mathcal{Z}]^{n, n}=0 \text { in } H^{n}\left(\mathcal{Y}_{U}, \Omega_{\mathcal{Y}_{U}}^{n}\right), \quad[\mathcal{Z}]_{t}^{n, n}=0 \text { in } H^{n}\left(\mathcal{Y}_{t}, \Omega_{\mathcal{Y} \mid \mathcal{Y}_{t}}^{n}\right), t \in U
$$

In [Voi94b], [Voi94a] (see also [Voi03, 5.2.2]), it was furthermore explained how to study the nonvanishing of the class $[\mathcal{Z}]^{n, n}$ by considering the "Leray filtration" on $\Omega_{\mathcal{Y}}^{n}$ :

$$
L^{s} \Omega_{\mathcal{Y}}^{n}:=\pi^{*} \Omega_{B}^{s} \wedge \Omega_{\mathcal{Y}}^{n-s}
$$

The associated graded vector bundle is

$$
G r_{L}^{s} \Omega_{\mathcal{Y}}^{n}=\pi^{*} \Omega_{B}^{s} \otimes \Omega_{\mathcal{Y} / B}^{n-s}
$$

It thus follows that there is a spectral sequence abutting to $R^{n} \pi_{*} \Omega_{\mathcal{Y}}^{n}$ :

$$
E_{1}^{s, q}\left(\Omega_{\mathcal{Y}}^{n}, L\right)=\Omega_{B}^{s} \otimes R^{s+q} \pi_{*} \Omega_{\mathcal{Y} / B}^{n-s} \Rightarrow R^{s+q} \pi_{*} \Omega_{\mathcal{Y}}^{n} .
$$

Of course, if $B$ is affine, we have

$$
H^{n}\left(\mathcal{Y}, \Omega_{\mathcal{Y}}^{n}\right)=H^{0}\left(B, R^{n} \pi_{*} \Omega_{\mathcal{Y}}^{n}\right)
$$

and thus the vanishing of $[\mathcal{Z}]^{n, n}$ is equivalent to the vanishing of $[\mathcal{Z}]_{t}^{n, n}$ for any $t \in B$.

One verifies (see [Voi03, 5.1]), as a consequence of the Katz-Oda description of the GaussManin connection, that the first differentials $d_{1}$ are the Griffiths $\bar{\nabla}$-maps induced by the GaussManin connection and Griffiths transversality:

$$
d_{1}=\bar{\nabla}_{s, n-s, s+q}: \Omega_{B}^{s} \otimes \mathcal{H}^{n-s, s+q} \rightarrow \Omega_{B}^{s+1} \otimes \mathcal{H}^{n-s-1, s+q+1},
$$

where the bundles $\mathcal{H}^{p, q}=R^{q} \pi_{*} \Omega_{\mathcal{Y} / B}^{p}$ are the Hodge bundles whose fibers at $t \in B$ are the spaces $H^{q}\left(\mathcal{Y}_{t}, \Omega_{\mathcal{Y}_{t}}^{p}\right)$.

This spectral sequence degenerates at $E_{2}$ (see [Ike03], [EP94]), as it is the case for the topological Leray spectral sequence, and by the same argument as in [Del68].

Assume now that our cycle $\mathcal{Z}$ has its class in $L^{s} H^{n}\left(\mathcal{Y}, \Omega_{\mathcal{Y}}^{n}\right)$, which is equivalent, if $B$ is affine and small enough, to the fact that $[\mathcal{Z}]_{t}^{n, n}$ belongs to the subspace $L^{s} H^{n}\left(\mathcal{Y}_{t}, \Omega_{\mathcal{Y}}^{n} \mathcal{Y}_{t}\right)$ for any $t \in B$, since we then have the base change property for the sheaf $R^{n} \pi_{*} \Omega_{\mathcal{Y}}^{n}$ endowed with the $L$-filtration and the formula

$$
L^{s} H^{n}\left(\mathcal{Y}, \Omega_{\mathcal{Y}}^{n}\right)=\Gamma\left(B, L^{s} R^{n} \pi_{*} \Omega_{\mathcal{Y}}^{n}\right)
$$




\section{Claire Voisin}

Our cycle then has an "infinitesimal invariant" (see [Voi94a], [Voi03, 5.2.2])

$$
\begin{array}{r}
\delta[\mathcal{Z}] \in \Gamma\left(B, E_{\infty}^{s, n-s}\left(\Omega_{\mathcal{Y}}^{n}, L\right)\right)=\Gamma\left(B, E_{2}^{s, n-s}\left(\Omega_{\mathcal{Y}}^{n}, L\right)\right) \\
=\Gamma\left(B, \operatorname{Ker} \bar{\nabla}_{s, n-s, n} / \operatorname{Im} \bar{\nabla}_{s-1, n-s+1, n-1}\right),
\end{array}
$$

where

$$
\bar{\nabla}_{s, n-s, n}: \Omega_{B}^{s} \otimes \mathcal{H}^{n-s, n} \rightarrow \Omega_{B}^{s+1} \otimes \mathcal{H}^{n-s-1, n+1}
$$

and

$$
\bar{\nabla}_{s-1, n-s+1, n-1}: \Omega_{B}^{s-1} \otimes \mathcal{H}^{n-s+1, n-1} \rightarrow \Omega_{B}^{s} \otimes \mathcal{H}^{n-s, n}
$$

are the $\bar{\nabla}$-maps of $(2.4)$.

Let $U \subset B$ be the dense Zariski open set where the coherent sheaf $E_{\infty}^{s, n-s, n}$ is locally free. The nonvanishing of the restricted infinitesimal invariant

$$
\delta[\mathcal{Z}]_{t} \in \operatorname{Ker} \bar{\nabla}_{s, n-s, n, t} / \operatorname{Im} \bar{\nabla}_{s-1, n-s+1, n-1, t}
$$

at some point $t \in U$ implies that the class $[\mathcal{Z}]^{n, n}$ is nonzero on any dense Zariski open subset of $U$, and thus by Lemma 2.2, the cycle $\mathcal{Z}_{t^{\prime}}$ is not rationally equivalent to 0 at a very general point $t^{\prime} \in B$.

Let us comment on the assumption made on $\mathcal{Z}^{n, n}$, namely that it belongs to $L^{s} H^{n}\left(\mathcal{Y}, \Omega_{\mathcal{Y}}^{n}\right)$, and relate it to the assumption made in Theorem 1.1 that $[\mathcal{Z}] \in L^{s} H^{2 n}(\mathcal{Y}, \mathbb{Q})$.

Proposition 2.3. Let $\mathcal{Z}$ be a codimension $n$ cycle on $\mathcal{Y}$ such that the Betti cohomology class $[\mathcal{Z}]$ belongs to $L^{s} H^{2 n}(\mathcal{Y}, \mathbb{Q})$. Then, after shrinking the base $B$ if necessary, the Dolbeault cycle class $[\mathcal{Z}]^{n, n}$ belongs to $L^{s} H^{n}\left(\mathcal{Y}, \Omega_{\mathcal{Y}}^{n}\right)$.

Proof. We use the fact, which is a consequence of the global invariant cycles theorem of Deligne (see [Del71], [Voi03, 4.3.3]), that there exist Hodge classes $\delta_{i}$ of degree $2 d$ with $d:=\operatorname{dim} \mathcal{Y} / B$ on $\mathcal{Y} \times_{B} \mathcal{Y}$, (where by Hodge classes, we mean Hodge classes in the pure part $W_{\text {pure }} H^{2 d}\left(\mathcal{Y} \times_{B} \mathcal{Y}, \mathbb{Q}\right)$ ) that act as Künneth projectors, that is, the induced morphisms $\delta_{i *}: R \pi_{*} \mathbb{Q} \rightarrow R \pi_{*} \mathbb{Q}$ factor as

$$
R \pi_{*} \mathbb{Q} \rightarrow R^{i} \pi_{*} \mathbb{Q}[-i] \rightarrow R \pi_{*} \mathbb{Q},
$$

where both maps induce the identity on cohomology of degree $i$ (and 0 in the other degrees). Furthermore, the sum $\sum_{i} \delta_{i}$ is equal to the class of the relative diagonal.

When the fibers $\mathcal{Y}_{t}$ have the property, as it is the case for an abelian fibration, that the Künneth components of their diagonals are algebraic, thus providing cycles $\Delta_{i, t}$ on $\mathcal{Y}_{t} \times \mathcal{Y}_{t}$ with classes the $i$-th Künneth projectors $\delta_{i, t} \in H^{2 d}\left(\mathcal{Y}_{t} \times \mathcal{Y}_{t}, \mathbb{Q}\right)$, we can construct the $\delta_{i}$ 's over a generically finite cover $B^{\prime}$ of $B$, by spreading out the $\Delta_{i, t}$ (which needs to make a base change) and taking the cohomology class $\delta_{i}$ of the spread-out cycles $\Delta_{i}$.

We now let the $\delta_{i}$ act on the classes $[\mathcal{Z}]$ and $[\mathcal{Z}]^{n, n}$. For the second action, we use the fact that $\delta_{i}$ is a Hodge class on $\mathcal{Y} \times_{B} \mathcal{Y}$, hence has a natural image in $H^{d}\left(\mathcal{Y} \times_{B} \mathcal{Y}, \Omega_{\mathcal{Y} \times{ }_{B}}^{d} \mathcal{Y}\right)$. As we have $\sum_{i} \delta_{i}=\left[\Delta_{\mathcal{Y} / B}\right]$, we get

$$
\sum_{i} \delta_{i *}=\operatorname{Id}: H^{2 n}(\mathcal{Y}, \mathbb{Q}) \rightarrow H^{2 n}(\mathcal{Y}, \mathbb{Q}) .
$$

Note also that the actions $\delta_{i *}$ are compatible with the Leray filtration on $H^{2 n}(\mathcal{Y}, \mathbb{Q})$ and the filtration $L$ on $H^{n}\left(\mathcal{Y}, \Omega_{\mathcal{Y}}^{n}\right)$. Looking at the way the $\delta_{i}$ operate on the spectral sequence associated to the filtration $L$ on $\Omega_{\mathcal{Y}}^{n}$, one finds that

$$
\delta_{i *}[\mathcal{Z}]^{n, n} \in L^{2 n-i} H^{n}\left(\mathcal{Y}, \Omega_{\mathcal{Y}}^{n}\right) .
$$




\section{INFINITESIMAL INVARIANTS AND 1-CYCLES IN JACOBIANS}

Using (2.6), we now write

$$
[\mathcal{Z}]=\sum_{i} \delta_{i *}[\mathcal{Z}]
$$

By Lemma 2.1, we get that if the Hodge class $\delta_{i *}[\mathcal{Z}]$ vanishes in $H^{2 n}(\mathcal{Y}, \mathbb{Q})$, then its Dolbeault counterpart $\delta_{i *}[\mathcal{Z}]^{n, n}$ vanishes in $H^{n}\left(\mathcal{Y}, \Omega_{\mathcal{Y}}^{n}\right)$.

The assumption that $[\mathcal{Z}] \in L^{s} H^{2 n}(\mathcal{Y}, \mathbb{Q})$ is equivalent to the assumption that $\delta_{i *}[\mathcal{Z}]=0$ for $i>2 n-s$, because $\operatorname{Im} \delta_{i *}: H^{2 n}(\mathcal{Y}, \mathbb{Q}) \rightarrow H^{2 n}(\mathcal{Y}, \mathbb{Q})$ is a direct summand contained in $L^{2 n-i} H^{2 n}(\mathcal{Y}, \mathbb{Q})$ and isomorphic to $G r_{L}^{2 n-i} H^{2 n}(\mathcal{Y}, \mathbb{Q})$. But then $\delta_{i *}[\mathcal{Z}]^{n, n}$ vanishes for $i>2 n-s$, that is,

$$
[\mathcal{Z}]^{n, n}=\sum_{i} \delta_{i *}[\mathcal{Z}]^{n, n}=\sum_{i \leqslant 2 n-s} \delta_{i *}[\mathcal{Z}]^{n, n} \in \sum_{s \leqslant 2 n-i} L^{2 n-i} H^{n}\left(\mathcal{Y}, \Omega_{\mathcal{Y}}^{n}\right)=L^{s} H^{n}\left(\mathcal{Y}, \Omega_{\mathcal{Y}}^{n}\right)
$$

The name "infinitesimal invariant" comes from the fact, proved in [Voi88], that for $s=1$, the infinitesimal invariant is the Griffiths infinitesimal invariant (cf. [Gri83]) obtained by differentiating the normal function associated to $\mathcal{Z}$.

\subsection{Infinitesimal invariants for cycles modulo algebraic equivalence}

Our main result in this section is the construction of an infinitesimal invariant for cycles modulo algebraic equivalence and the proof of Theorem 1.1. Let $\pi: \mathcal{Y} \rightarrow B$ be a smooth projective morphism and let $\mathcal{Z} \subset \mathcal{Y}$ be a cycle of codimension $n$. Note that Lemma 2.2 is not true for cycles modulo algebraic equivalence. However, we have Proposition 2.4 below, which will allow us to extract from the $\delta[\mathcal{Z}]$ defined in previous section an infinitesimal invariant associated to cycles modulo algebraic equivalence.

We will use the fact that the maps

$$
\bar{\nabla}_{s, n-s, n}: \Omega_{B}^{s} \otimes \mathcal{H}^{n-s, n} \rightarrow \Omega_{B}^{s+1} \otimes \mathcal{H}^{n-s-1, n+1}
$$

satisfy the relation

$$
\bar{\nabla}_{s, n-s, n}(\alpha \wedge \sigma)=-\alpha \wedge \bar{\nabla}_{s-1, n-s, n}(\sigma)
$$

for $\alpha$ a section of $\Omega_{B}, \sigma$ a section of $\Omega_{B}^{s-1} \otimes \mathcal{H}^{n-s, n}$. This is proved as follows: for $\alpha, \sigma$ as above, let $\tilde{\sigma}$ be a section of $F^{n-s} \mathcal{H}^{2 n-s}$ which lifts $\sigma$. Then by definition,

$$
\bar{\nabla}_{s, n-s, n}(\alpha \wedge \sigma)=\nabla(\alpha \wedge \tilde{\sigma}) \quad \bmod \Omega_{B}^{s+1} \otimes F^{n} \mathcal{H}^{2 n-s} .
$$

By the Leibniz rule, this is also equal to $-\alpha \wedge \nabla \tilde{\sigma}+d \alpha \wedge \tilde{\sigma} \bmod \Omega_{B}^{s+1} \otimes F^{n} \mathcal{H}^{2 n-s}$. As $d \alpha \wedge \tilde{\sigma}$ is a section of $\Omega_{B}^{s+1} \otimes F^{n} \mathcal{H}^{2 n-s}$, we get

$$
\bar{\nabla}_{s, n-s, n}(\alpha \wedge \sigma)=-\alpha \wedge \nabla \tilde{\sigma} \bmod . \Omega_{B}^{s+1} \otimes F^{n} \mathcal{H}^{2 n-s} .
$$

It follows from (2.7) that

$$
\Omega_{B} \wedge \operatorname{Ker} \bar{\nabla}_{s-1, n-s, n} \subset \operatorname{Ker} \bar{\nabla}_{s, n-s, n},
$$

as implicitly stated in Theorem 1.1. We now have the following result.

Proposition 2.4. Assume that for any $t \in B$, (a multiple of) $\mathcal{Z}_{t}$ is algebraically equivalent to 0 and that furthermore $[\mathcal{Z}]^{n, n} \in L^{s} H^{n}\left(\mathcal{Y}, \Omega_{\mathcal{Y}}^{n}\right)$. Then there is a Zariski open set $U \subset B$ such that over $U$, the infinitesimal invariant $\delta[\mathcal{Z}]^{n, n}$ belongs to the image of $\Omega_{B} \wedge \operatorname{Ker} \bar{\nabla}_{s-1, n-s, n}$ in

$$
\frac{\operatorname{Ker} \bar{\nabla}_{s, n-s, n}}{\operatorname{Im} \bar{\nabla}_{s-1, n-s+1, n-1}} \text {. }
$$




\section{Claire Voisin}

The following lemma is useful as it explains the simple form that the space of infinitesimal invariants in Theorem 1.1 takes.

Lemma 2.5. For $s \geqslant 2$, the space $\Omega_{B} \wedge \operatorname{Ker} \bar{\nabla}_{s-1, n-s, n} \subset \operatorname{Ker} \bar{\nabla}_{s, n-s, n}$ contains $\operatorname{Im} \bar{\nabla}_{s-1, n-s+1, n-1}$. It follows that the quotient

$$
\frac{\operatorname{Ker} \bar{\nabla}_{s-1, n-s, n}}{\operatorname{Im} \bar{\nabla}_{s-1, n-s+1, n-1}+\Omega_{B} \wedge \operatorname{Ker} \bar{\nabla}_{s-1, n-s, n}}
$$

is equal to

$$
\frac{\operatorname{Ker} \bar{\nabla}_{s-1, n-s, n}}{\Omega_{B} \wedge \operatorname{Ker} \bar{\nabla}_{s-1, n-s, n}} .
$$

Proof. Indeed, for $s \geqslant 2$, we have $s-1 \geqslant 1$ and thus for a section $\eta$ of $\Omega_{B}^{s-1} \otimes \mathcal{H}^{n-s+1, n-1}$, we can write, locally, $\eta=\sum_{i} \alpha_{i} \wedge \eta_{i}$, where the $\alpha_{i}$ are sections of $\Omega_{B}$ and the $\eta_{i}$ are sections of $\Omega_{B}^{s-2} \otimes \mathcal{H}^{n-s+1, n-1}$. By $(2.7)$, we have

$$
\bar{\nabla}_{s-1, n-s+1, n-1}(\eta)=-\sum_{i} \alpha_{i} \wedge \bar{\nabla}_{s-2, n-s+1, n-1}\left(\eta_{i}\right) .
$$

This proves the result since $\bar{\nabla}_{s-2, n-s+1, n-1}\left(\eta_{i}\right) \in \operatorname{Ker} \bar{\nabla}_{s-1, n-s, n}$.

The proof of Proposition 2.4 is postponed to the end of the section. We first show how it implies Theorem 1.1.

Proof of Theorem 1.1. Starting from a cycle $\mathcal{Z}$ with class $[\mathcal{Z}] \in L^{s} H^{2 n}(\mathcal{Y}, \mathbb{Q})$, we know by Proposition 2.3 that after shrinking $B$, if necessary, the class $[\mathcal{Z}]^{n, n}$ belongs to $L^{s} H^{n}\left(\mathcal{Y}, \Omega_{\mathcal{Y}}^{n}\right)$. We thus have the infinitesimal invariant $\delta[\mathcal{Z}]$ and its restrictions $\delta[\mathcal{Z}]_{t} \in G r_{L}^{s} H^{n}\left(\mathcal{Y}_{t}, \Omega_{\mathcal{Y} \mid \mathcal{Y}_{t}}^{n}\right)$ for any point $t \in B$. Let $U$ be the dense Zariski open subset of $B$ where the sheaf

$$
\frac{\operatorname{Ker} \bar{\nabla}_{s, n-s, n}}{\Omega_{B} \wedge \operatorname{Ker} \bar{\nabla}_{s-1, n-s, n}+\operatorname{Im} \bar{\nabla}_{s-1, n-s-1, n-1}}
$$

is locally free. Then the nonvanishing of the infinitesimal invariant

$$
\delta[\mathcal{Z}]_{t} \in \frac{\operatorname{Ker} \bar{\nabla}_{s, n-s, n, t}}{\operatorname{Im} \bar{\nabla}_{s-1, n-s-1, n-1, t}}
$$

modulo the image of $\Omega_{B, t} \otimes \operatorname{Ker} \bar{\nabla}_{s-1, n-s, n, t}$ under the wedge product map implies that for any dense Zariski open subset $U^{\prime} \subset U$,

$$
\delta[\mathcal{Z}]_{\mid U^{\prime}} \in \Gamma\left(U^{\prime}, \frac{\operatorname{Ker} \bar{\nabla}_{s, n-s, n}}{\operatorname{Im} \bar{\nabla}_{s-1, n-s-1, n-1}}\right)
$$

does not vanish modulo

$$
\operatorname{Im}\left[\Gamma\left(U^{\prime}, \Omega_{U^{\prime}} \wedge \operatorname{Ker} \bar{\nabla}_{s-1, n-s, n}\right) \rightarrow \Gamma\left(U^{\prime}, \frac{\operatorname{Ker} \bar{\nabla}_{s, n-s, n}}{\operatorname{Im} \bar{\nabla}_{s-1, n-s-1, n-1}}\right)\right],
$$

hence it follows from Proposition 2.4 that the cycle $\mathcal{Z}_{t}$ is not algebraically equivalent to 0 for very general $t \in U$.

For $s \geqslant 2$, we can apply Lemma 2.5. Denoting then by $\delta[Z]_{\text {alg }}$ and $\delta[\mathcal{Z}]_{t, a l g}$ the image of $\delta[\mathcal{Z}]$ and $\delta[\mathcal{Z}]_{t}$ in

$$
\Gamma\left(U, \frac{\operatorname{Ker} \bar{\nabla}_{s, n-s, n}}{\Omega_{U} \wedge \operatorname{Ker} \bar{\nabla}_{s-1, n-s, n}}\right) \quad \text { and } \frac{\operatorname{Ker} \bar{\nabla}_{s, n-s, n, t}}{\Omega_{B, t} \wedge \operatorname{Ker} \bar{\nabla}_{s-1, n-s, n, t}},
$$




\section{INFINITESIMAL INVARIANTS AND 1-CYCLES IN JACOBIANS}

respectively, we have constructed the desired infinitesimal invariant for cycles modulo algebraic equivalence satisfying the conclusion of Theorem 1.1 and Corollary 1.2.

Let us first make a few remarks.

Remark 2.6. In [Nor93], Nori uses the cycle class $[\mathcal{Z}]^{n, n}$ to conclude that a certain cycle is not algebraically equivalent to 0 at a general point $t \in B$, assuming its cohomology class $\mathcal{Z}$ does not vanish on the total space. However, he does not introduce the infinitesimal invariant above (although the infinitesimal computation is hidden in his arguments), for the following reason. In his situation, the kernel of the map

$$
\bar{\nabla}_{s-1, n-s, n}: \Omega_{B}^{s-1} \otimes \mathcal{H}^{n-s, n} \rightarrow \Omega_{B}^{s} \otimes \mathcal{H}^{n-s-1, n+1}
$$

is equal to the image of the map

$$
\bar{\nabla}_{s-2, n-s+1, n-1}: \Omega_{B}^{s-2} \otimes \mathcal{H}^{n-s+1, n-1} \rightarrow \Omega_{B}^{s-1} \otimes \mathcal{H}^{n-s, n},
$$

and it thus follows that

$$
\Omega_{B} \wedge \operatorname{Ker} \bar{\nabla}_{s-1, n-s, n} \subset \operatorname{Im}\left(\bar{\nabla}_{s-1, n-s+1, n-1}: \Omega_{B}^{s-1} \otimes \mathcal{H}^{n-s+1, n-1} \rightarrow \Omega_{B}^{s} \otimes \mathcal{H}^{n-s, n}\right) .
$$

This is why Nori can use the previously defined invariant $\delta[\mathcal{Z}]$ (see Section 2.1) to deal with cycles modulo algebraic equivalence. The same remark applies to the paper of Fakhruddin who uses similar methods applied to general abelian varieties.

Our second remark concerns the consistency of the existence of the above defined invariant and the conjecture made in [Voi04]. We first recall what is this conjecture: Here we can consider for the Bloch-Beilinson filtration on Chow groups with rational coefficients any of the filtrations mentioned in the introduction, for example the one defined by Shuji Saito in [Sai96], or, in the case of an abelian variety, the one which is induced by the Beauville decomposition as in (1.1). Let us denote such a filtration by $F$. These filtrations are conjectured to satisfy the crucial property that

$$
F^{k+1} \mathrm{CH}^{k}(Y)_{\mathbb{Q}}=0 .
$$

These filtrations also induce similar filtrations on Chow groups modulo algebraic equivalence.

In [Voi04] we made (and explored the consequences of) the following conjecture (which generalizes Nori's conjecture [Nor93] on $\left.\mathrm{CH}^{2}\right)$.

Conjecture 2.7. We have

$$
F^{k} \mathrm{CH}^{k}(Y) / a l g=0 .
$$

We now want to observe the following fact, which is consistent with this conjecture.

Lemma 2.8. Let $\mathcal{Z} \subset \mathcal{Y} \rightarrow B$ be a cycle of codimension $n$ such that $[\mathcal{Z}] \in L^{n} H^{n}\left(\mathcal{Y}, \Omega_{\mathcal{Y}}^{n}\right)$. Then for any $t \in B$, the infinitesimal invariant

$$
\delta[\mathcal{Z}]_{t, a l g} \in \frac{\operatorname{Ker} \bar{\nabla}_{n, 0, n, t}}{\Omega_{B, t} \wedge \operatorname{Ker} \bar{\nabla}_{n-1,0, n, t}}
$$

vanishes.

Proof. This is obvious because the group we are looking at is the following (assuming $n \geqslant 2$, see Lemma 2.5):

$$
\frac{\operatorname{Ker}\left(\bar{\nabla}_{n, 0, n}: \Omega_{B, t}^{n} \otimes H^{0, n}\left(\mathcal{Y}_{t}\right) \rightarrow \Omega_{B_{t}}^{n+1} \otimes H^{-1, n+1}\left(\mathcal{Y}_{t}\right)\right)}{\Omega_{B, t} \wedge \operatorname{Ker}\left(\bar{\nabla}_{n-1,0, n}: \Omega_{B, t}^{n-1} \otimes H^{0, n}\left(\mathcal{Y}_{t}\right) \rightarrow \Omega_{B, t}^{n} \otimes H^{-1, n+1}\left(\mathcal{Y}_{t}\right)\right)} .
$$




\section{Claire Voisin}

Hence, because $H^{-1, n+1}\left(\mathcal{Y}_{t}\right)=0$, the $\bar{\nabla}$-maps vanish in this range, and our group is in fact

$$
\frac{\Omega_{B, t}^{n} \otimes H^{0, n}\left(\mathcal{Y}_{t}\right)}{\Omega_{B, t} \wedge \Omega_{B, t}^{n-1} \otimes H^{0, n}\left(\mathcal{Y}_{t}\right)},
$$

which is 0 because $n>0$.

Our last remark concerns the assumption that $[\mathcal{Z}] \in L^{s} H^{2 n}(\mathcal{Y}, \mathbb{Q})$ and will be useful for our main application. There are two cases where the Leray level of an algebraic cycle in a family of varieties is easy to compute:

1) The family $\mathcal{Y}$ is a family of smooth ample hypersurfaces or complete intersections in a projective space (or more generally, any ambient variety $X$ whose rational cohomology is made of classes of algebraic cycles). Then the hard Lefschetz theorem in this case says that the interesting part of the cohomology of $\mathcal{Y}_{t}$ is supported in degree $m:=\operatorname{dim} \mathcal{Y}_{t}$. Given any cycle $\mathcal{Z}$ of codimension $n<\operatorname{dim} \mathcal{Y}_{t} / 2$ in $\mathcal{Y}$, we can write (at least with rational coefficients)

$$
\mathcal{Z}=\mathcal{Z}^{\prime}+\Gamma
$$

where $\Gamma$ is the restriction of a cycle coming from $X$ and $\mathcal{Z}_{\mid \mathcal{Y}_{t}}^{\prime}$ is cohomologous to 0 . Looking more precisely at the shape of the Leray spectral sequence of $\mathcal{Y} \rightarrow B$ and applying the Lefschetz hyperplane section theorem, one finds that on an adequate dense Zariski open set $U \subset B$, the Dolbeault cohomology class of $\mathcal{Z}_{\mid \mathcal{Y}_{U}}^{\prime}$ belongs to $L^{2 n-d} H^{2 n}\left(\mathcal{Y}_{U}, \mathbb{Q}\right)$, where $d=\operatorname{dim} \mathcal{Y}_{t}$.

2) The second case where the Leray level is easy to compute is the case of a cycle in a family of abelian varieties $\mathcal{A} \rightarrow B$. In this case we have the following fact.

Lemma 2.9. If the cycle $\mathcal{Z}$ satisfies the condition that

$$
\mathcal{Z}_{t} \in \mathrm{CH}^{n}\left(\mathcal{A}_{t}\right)_{s}
$$

for any $t \in B$, then there is a dense Zariski open set $U \subset B$ such that $[\mathcal{Z}]_{U} \in L^{s} H^{2 n}\left(\mathcal{A}_{U}, \mathbb{Q}\right)$.

Proof. Indeed, under our assumptions, Lemma 2.2 and the relative Beauville decomposition (see [DM91]) show that there exists a dense Zariski open set $U \subset B$ such that for any $k \in \mathbb{Z}^{*}$,

$$
\mu_{k}^{*} \mathcal{Z}_{U}=k^{2 n-s} \mathcal{Z} \text { in } \mathrm{CH}^{n}\left(\mathcal{A}_{U}\right) \text {. }
$$

It thus follows that for any $k$, the class $[\mathcal{Z}] \in H^{2 n}\left(\mathcal{A}_{U}, \mathbb{Q}\right)$ satisfies

$$
\mu_{k}^{*}[\mathcal{Z}]=k^{2 n-s}[\mathcal{Z}] \text { in } H^{2 n}\left(\mathcal{A}_{U}, \mathbb{Q}\right) .
$$

But there is a canonical Deligne decomposition

$$
R \pi_{*} \mathbb{Q}=\oplus R^{i} \pi_{*} \mathbb{Q}[-i]
$$

such that for any $i$,

$$
\mu_{k}^{*} \circ \pi_{i}=k^{i} \pi_{i}: R \pi_{*} \mathbb{Q} \rightarrow R \pi_{*} \mathbb{Q},
$$

where the $\pi_{i}$ are the projectors associated to the decomposition (2.11) (cf. [DM91]). It follows that

$$
L^{s} H^{2 n}\left(\mathcal{A}_{U}, \mathbb{Q}\right)=\oplus_{i \geqslant s} H^{2 n}\left(\mathcal{A}_{U}, \mathbb{Q}\right)_{i},
$$

where

$$
H^{2 n}\left(\mathcal{A}_{U}, \mathbb{Q}\right)_{i}=\left\{\alpha \in H^{2 n}\left(\mathcal{A}_{U}, \mathbb{Q}\right), \mu_{k}^{*}[\mathcal{Z}]=k^{2 n-i}[\mathcal{Z}] \text { in } H^{2 n}\left(\mathcal{A}_{U}, \mathbb{Q}\right) \text { for any } k\right\} .
$$

By (2.10) and (2.12), we therefore have $[\mathcal{Z}]_{U} \in L^{s} H^{2 n}\left(\mathcal{A}_{U}, \mathbb{Q}\right)$. 


\section{INFINITESIMAL INVARIANTS AND 1-CYCLES IN JACOBIANS}

Proof of Proposition 2.4. We first prove the result in the case which will be useful for us, namely when $\pi: \mathcal{Y} \rightarrow B$ is a family of abelian varieties. The proof is easier in this case because, as the proof of Lemma 2.9 shows, not only does the spectral sequence abutting to $R^{n} \pi_{*}\left(\mathcal{Y}, \Omega_{\mathcal{Y}}^{n}\right)$ degenerate at $E_{2}$, there is also a natural decomposition

$$
R^{n} \pi_{*} \Omega_{\mathcal{Y}}^{n}=\oplus_{s}\left(R^{n} \pi_{*} \Omega_{\mathcal{Y}}^{n}\right)_{s},
$$

where

$$
\left(R^{n} \pi_{*} \Omega_{\mathcal{Y}}^{n}\right)_{s}=\left\{\alpha \in R^{n} \pi_{*} \Omega_{\mathcal{Y}}^{n}, \mu_{k}^{*} \alpha=k^{2 n-s} \alpha, \forall k \in \mathbb{Z}\right\}
$$

and the decomposition (2.13) is a splitting of the filtration induced by the filtration $L$ on $\Omega \mathcal{Y}$. We will explain at the end of the proof how to modify the argument to make it work in general.

So assume that $\mathcal{Y} \rightarrow B$ is a family of abelian varieties over a smooth affine variety $B$, and consider a codimension $n$ cycle $\mathcal{Z}$ on $\mathcal{Y}$. Assume that for any $t \in B, \mathcal{Z}_{t}$ is algebraically equivalent to 0 , and that furthermore $[\mathcal{Z}] \in L^{s} H^{2 n}(\mathcal{Y}, \mathbb{Q})$. Using the relative Beauville decomposition of Deninger-Murre [DM91], we can replace $\mathcal{Z}$ by $\mathcal{Z}_{s}$, which does not change the class $\delta[\mathcal{Z}] \in G r_{s}^{L} H^{n}\left(\mathcal{Y}, \Omega_{\mathcal{Y}}^{n}\right)$ and preserves the assumption that its restrictions to the fibers is algebraically equivalent to 0 . So from now on we assume that $\mathcal{Z}=\mathcal{Z}_{s}$.

The definition of algebraic equivalence together with an elementary argument involving countability of Chow varieties and uncountability of $\mathbb{C}$ implies that over a Zariski dense open subset $U$ of $B$, there exist

(i) a family of smooth nonnecessarily connected curves $\rho: \mathcal{C} \rightarrow U$;

(ii) a divisor $\mathcal{D}$ of $\mathcal{C}$ which is homologous to 0 on the fibers of $\rho$;

(iii) a codimension $n$ correspondence $\Gamma \subset \mathcal{C} \times{ }_{U} \mathcal{Y}_{U}$ with $\mathbb{Q}$-coefficients,

such that

$$
\mathcal{Z}=\Gamma_{*}(\mathcal{D}) \text { in } \mathrm{CH}^{n}\left(\mathcal{Y}_{U}\right) .
$$

Here we recall that, denoting by $p_{1}$ and $p_{2}$ the two proper smooth projections from $\mathcal{C} \times_{U} \mathcal{Y}_{U}$ to $\mathcal{C}$ and $\mathcal{Y}_{U}$, respectively,

$$
\Gamma_{*}(\mathcal{D}):=p_{2 *}\left(p_{1}^{*} \mathcal{D} \cdot \Gamma\right) .
$$

Using the natural functoriality properties of the Dolbeault cycle class, the equality of cycles (2.14) now provides the corresponding equality of Dolbeault cohomology classes:

$$
\left[\mathcal{Z}_{U}\right]^{n, n}=p_{2 *}\left([\Gamma]^{n, n} \cdot p_{1}^{*}[\mathcal{D}]^{1,1}\right) \text { in } H^{n}\left(\mathcal{Y}_{U}, \Omega_{\mathcal{Y}_{U}}^{n}\right)
$$

We now again use the relative Beauville decomposition for cycles in the family $\mathcal{C} \times_{U} \mathcal{Y} \rightarrow \mathcal{C}$. The cycle $\Gamma$ decomposes as

$$
\Gamma=\sum_{p} \Gamma_{p}
$$

with $\mu_{k}^{*} \Gamma_{p}=k^{2 n-p} \Gamma_{p}$. Accordingly, the class

$$
[\Gamma]^{n, n} \in H^{n}\left(\mathcal{C} \times_{U} \mathcal{Y}_{U}, \Omega_{\mathcal{C} \times_{U}}^{n} \mathcal{Y}_{U}\right)
$$

decomposes as

$$
[\Gamma]^{n, n}=\bigoplus_{p}\left[\Gamma_{p}\right]^{n, n}
$$

where $\left[\Gamma_{p}\right]^{n, n} \in H^{n}\left(\mathcal{C} \times_{U} \mathcal{Y}_{U}, \Omega_{\mathcal{C} \times_{U}}^{n} \mathcal{Y}_{U}\right)$ satisfies the condition

$$
\mu_{k}^{*}\left[\Gamma_{p}\right]^{n, n}=k^{2 n-p}\left[\Gamma_{p}\right]^{n, n}, \forall k \in \mathbb{Z}^{*} .
$$




\section{Claire Voisin}

Recall that $[\mathcal{Z}]_{U}^{n, n}$ belongs to $H^{n}\left(\mathcal{Y}_{U}, \Omega_{\mathcal{Y}_{U}}^{n}\right)_{s}$. Observing that the maps $\mu_{k}^{*}$ are compatible with the pushforward map $p_{2 *}$, in the sense that

$$
p_{2 *} \circ \mu_{k}^{*}=\mu_{k}^{*} \circ p_{2 *},
$$

we get the following equality from (2.15), (2.16) and (2.17):

$$
\left[\mathcal{Z}_{U}\right]^{n, n}=p_{2 *}\left(\left[\Gamma_{s}\right]^{n, n} \cdot p_{1}^{*}[\mathcal{D}]^{1,1}\right) \text { in } H^{n}\left(\mathcal{Y}_{U}, \Omega_{\mathcal{Y}_{U}}^{n}\right)_{s} \subset L^{s} H^{n}\left(\mathcal{Y}_{U}, \Omega_{\mathcal{Y}_{U}}^{n}\right)
$$

Projecting everything modulo $L^{s+1}$, we get

$$
\delta[\mathcal{Z}]=p_{2 *}\left(\delta\left[\Gamma_{s}\right] \cdot p_{1}^{*} \delta[\mathcal{D}]\right) \text { in } G r_{L}^{s} H^{n}\left(\mathcal{Y}_{U}, \Omega_{\mathcal{Y}_{U}}^{n}\right)=\Gamma\left(U, E_{2}^{s, n-s}\left(\Omega_{\mathcal{Y}_{U}}^{n}, L\right)\right) .
$$

Here $[\mathcal{D}]^{1,1} \in H^{1}\left(\mathcal{C}, \Omega_{\mathcal{C}}\right)$, and as $\mathcal{D}$ is cohomologous to 0 on the fibers $\mathcal{C}_{t}, t \in U$, we have $[\mathcal{D}] \in L^{1} H^{1}\left(\mathcal{C}, \Omega_{\mathcal{C}}\right)$, so that

$$
\delta[\mathcal{D}] \in \Gamma\left(U, \Omega_{U} \otimes \mathcal{H}_{\mathcal{C}}^{0,1} / \bar{\nabla}_{\mathcal{C}} \mathcal{H}_{\mathcal{C}}^{1,0}\right),
$$

where the bundles $\mathcal{H}_{\mathcal{C}}^{0,1}=R^{1} \rho_{*} \mathcal{O}_{\mathcal{C}}, \mathcal{H}_{\mathcal{C}}^{1,0}=R^{0} \rho_{*} \Omega_{\mathcal{C} / U}$ are the Hodge bundles of the family $\rho: \mathcal{C} \rightarrow U$ and $\nabla_{\mathcal{C}}, \bar{\nabla}_{\mathcal{C}}$ denote the corresponding Gauss-Manin connection and $\bar{\nabla}$-map. (To avoid confusion, below we will also write $\bar{\nabla} \mathcal{Y}, \mathcal{H}_{\mathcal{Y}}^{p, q}$ for the infinitesimal variation of Hodge structure of the family $\mathcal{Y}$ or $\mathcal{Y}_{U}$.) We will denote by $\widehat{\delta[\mathcal{D}]}$ any lift of $\delta[\mathcal{D}]$ in $\Omega_{U} \otimes \mathcal{H}_{\mathcal{C}}^{0,1}$.

We now examine

$$
\gamma:=\delta\left[\Gamma_{s}\right] \in H^{n}\left(\mathcal{C} \times_{U} \mathcal{Y}_{U}, \Omega_{\mathcal{C} \times_{U} \mathcal{Y}_{U}}^{n}\right)_{s}=H^{0}\left(U,\left(R^{n} \pi_{*}^{\prime} \Omega_{\mathcal{C} \times_{U}}^{n} \mathcal{Y}_{U}\right)_{s}\right)
$$

where $\pi^{\prime}:=\pi \circ p_{2}: \mathcal{C} \times_{U} \mathcal{Y}_{U} \rightarrow U$, and as before

$$
\left(R^{n} \pi_{*}^{\prime} \Omega_{\mathcal{C} \times_{U} \mathcal{Y}_{U}}^{n}\right)_{s}=\left\{\alpha \in R^{n} \pi_{*}^{\prime} \Omega_{\mathcal{C} \times_{U} \mathcal{Y}_{U}}^{n}, \mu_{k}^{*} \alpha=k^{2 n-s} \alpha\right\} .
$$

We recall that the class $\gamma$ is a section of

$$
\left(R^{n} \pi_{*}^{\prime} \Omega_{\mathcal{C} \times_{U}}^{n} \mathcal{Y}_{U}\right)_{s}
$$

with $\pi^{\prime}=\rho \circ p_{1}=\pi \circ p_{2}: \mathcal{C} \times_{U} \mathcal{Y}_{U} \rightarrow U$. Considering the exact sequence

$$
0 \rightarrow R^{1} \rho_{*}\left(R^{n-1} p_{1 *} \Omega_{\mathcal{C} \times_{U}}^{n} \mathcal{Y}_{U}\right) \rightarrow R^{n} \pi_{*}^{\prime} \Omega_{\mathcal{C} \times_{U}}^{n} \mathcal{Y}_{U} \rightarrow \rho_{*}\left(R^{n} p_{1 *} \Omega_{\mathcal{C} \times_{U}}^{n} \mathcal{Y}_{U}\right) \rightarrow 0,
$$

and taking the direct summand (where $\mu_{k}^{*}$ acts by multiplication by $k^{2 n-s}$ ), we get

$$
0 \rightarrow R^{1} \rho_{*}\left(R^{n-1} p_{1 *} \Omega_{\mathcal{C} \times_{U}}^{n} \mathcal{Y}_{U}\right)_{s} \rightarrow\left(R^{n} \pi_{*}^{\prime} \Omega_{\mathcal{C} \times_{U}}^{n} \mathcal{Y}_{U}\right)_{s} \rightarrow \rho_{*}\left(R^{n} p_{1 *} \Omega_{\mathcal{C} \times_{U} \mathcal{Y}_{U}}^{n}\right)_{s} \rightarrow 0 .
$$

As $\Omega_{\mathcal{C} \times_{U} \mathcal{Y}_{U}}^{n}$ projects naturally to $p_{1}^{*} \Omega_{\mathcal{C} / U} \otimes p_{2}^{*} \Omega_{\mathcal{Y}}^{n-1}$, we get a morphism

$$
\rho_{*}\left(R^{n} p_{1 *} \Omega_{\mathcal{C} \times_{U}}^{n} \mathcal{Y}_{U}\right)_{s} \rightarrow \mathcal{H}_{\mathcal{C}}^{1,0} \otimes\left(R^{n} \pi_{*} \Omega_{\mathcal{Y}_{U}}^{n-1}\right)_{s-1}
$$

where the subscript $s-1$ instead of $s$ on the right follows from formula (2.21) and the shift in total degree.

As was explained in Section 2.1, $\left(R^{n} \pi_{*} \Omega_{\mathcal{Y}_{U}}^{n-1}\right)_{s-1}$ is computed as

$$
\frac{\operatorname{Ker}\left(\bar{\nabla}_{\mathcal{Y}, s-1, n-s, n}: \Omega_{U}^{s-1} \otimes \mathcal{H}_{\mathcal{Y}}^{n-s, n} \rightarrow \Omega_{U}^{s} \otimes \mathcal{H}_{\mathcal{Y}}^{n-s-1, n+1}\right)}{\operatorname{Im}\left(\bar{\nabla}_{\mathcal{Y}, s-2, n-s+1, n-1}: \Omega_{U}^{s-2} \otimes \mathcal{H}_{\mathcal{Y}}^{n-s+1, n-1} \rightarrow \Omega_{U}^{s-1} \otimes \mathcal{H}_{\mathcal{Y}}^{n-s, n}\right)} .
$$

Combining these various morphisms and identifications, we find that $\gamma$ provides a class

$$
\gamma_{1} \in \Gamma\left(U, \mathcal{H}_{\mathcal{C}}^{1,0} \otimes \frac{\operatorname{Ker} \bar{\nabla}_{\mathcal{Y}, s-1, n-s, n}}{\operatorname{Im} \bar{\nabla}_{\mathcal{Y}, s-2, n-s+1, n-1}}\right) .
$$

We will denote by $\tilde{\gamma}_{1}$ any lift of $\gamma_{1}$ in $\Gamma\left(U, \mathcal{H}_{\mathcal{C}}^{1,0} \otimes \operatorname{Ker} \bar{\nabla}_{\mathcal{Y}, s-1, n-s, n}\right) \subset \Gamma\left(U, \mathcal{H}_{\mathcal{C}}^{1,0} \otimes \Omega_{U}^{s-1} \otimes \mathcal{H}_{\mathcal{Y}}^{n-s, n}\right)$. 


\section{INFINITESIMAL INVARIANTS AND 1-CYCLES IN JACOBIANS}

The proof of Proposition 2.4 in the case of a family of abelian varieties is then an immediate consequence of Lemma 2.10 below. Indeed, it says in particular that under the assumptions of Proposition 2.4,

$$
\delta[\mathcal{Z}] \in \frac{\operatorname{Ker} \bar{\nabla}_{\mathcal{Y}, s, n-s, n}}{\operatorname{Im} \bar{\nabla}_{\mathcal{Y}, s-1, n-s+1, n-1}}
$$

belongs to the image, under the wedge product map, of the sheaf

$$
\Omega_{U} \otimes \frac{\operatorname{Ker} \bar{\nabla}_{\mathcal{Y}, s-1, n-s, n}}{\operatorname{Im} \bar{\nabla}_{\mathcal{Y}, s-2, n-s+1, n-1}} .
$$

LEMma 2.10. With the same assumptions and notation as above, we have

$$
\delta[\mathcal{Z}]=w\left(<\widetilde{\delta[\mathcal{D}]}, \tilde{\gamma}_{1}>\right) \text { modulo } \operatorname{Im} \bar{\nabla}_{\mathcal{Y}, s-1, n-s+1, n-1},
$$

where the brackets mean that we use the duality between $\mathcal{H}_{\mathcal{C}}^{0,1}$ and $\mathcal{H}_{\mathcal{C}}^{1,0}$ to get a contraction map

$$
<,>:\left(\Omega_{U} \otimes \mathcal{H}_{\mathcal{C}}^{0,1}\right) \otimes\left(\mathcal{H}_{\mathcal{C}}^{1,0} \otimes \Omega_{U}^{s-1} \otimes \mathcal{H}_{\mathcal{Y}}^{n-s, n}\right) \rightarrow \Omega_{U} \otimes \Omega_{U}^{s-1} \otimes \mathcal{H}_{\mathcal{Y}}^{n-s, n},
$$

and the map $w$ is induced by the wedge product map $\Omega_{U} \otimes \Omega_{U}^{s-1} \rightarrow \Omega_{U}^{s}$.

Before giving the proof of (2.22), let us explain why the projection of

$$
w\left(<\widetilde{\delta[\mathcal{D}]}, \tilde{\gamma}_{1}>\right) \in \Gamma\left(U, \operatorname{Ker} \bar{\nabla}_{\mathcal{Y}, s, n-s, n}\right) \subset \Gamma\left(U, \Omega_{U}^{s} \otimes \mathcal{H}_{\mathcal{Y}}^{n-s, n}\right)
$$

in the quotient

$$
\frac{\operatorname{Ker} \bar{\nabla}_{\mathcal{Y}, s, n-s, n}}{\operatorname{Im} \bar{\nabla}_{\mathcal{Y}, s-1, n-s+1, n-1}}
$$

does not depend on the choice of the lifts $\widetilde{\delta[\mathcal{D}]}, \tilde{\gamma}_{1}$. If we change $\tilde{\gamma}_{1}$ by a $\bar{\nabla} \mathcal{Y}_{\text {-exact section of }}$ $\Omega_{U}^{s-1} \otimes \mathcal{H}_{\mathcal{C}}^{1,0} \otimes \mathcal{H}_{\mathcal{Y}}^{n-s, n}$, that is, $\left(\tilde{\gamma}_{1}\right)^{\prime}=\tilde{\gamma}_{1}+\bar{\nabla}_{\mathcal{Y}, s-2, n-s+1, n-1}\left(\gamma^{\prime}\right)$ for a section $\gamma^{\prime}$ of $\Omega_{U}^{s-2} \otimes \mathcal{H}_{\mathcal{C}}^{1,0} \otimes$ $\mathcal{H}_{\mathcal{Y}}^{n-s+1, n-1}$, then we clearly have

$$
w\left(<\delta[\widetilde{\mathcal{D}}],\left(\tilde{\gamma}_{1}\right)^{\prime}>=w\left(<\widetilde{\delta[\mathcal{D}]}, \tilde{\gamma}_{1}>\right)+\bar{\nabla}_{\mathcal{Y}, s-1, n-s+1, n-1}\left(w\left(<\delta[\widetilde{\mathcal{D}}], \gamma^{\prime}>\right)\right),\right.
$$

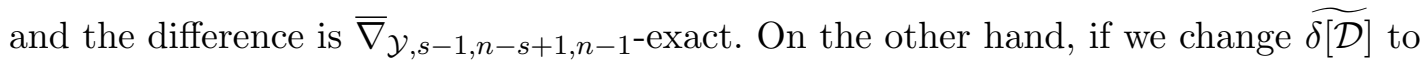

$$
(\widetilde{\delta[\mathcal{D}]})^{\prime}=\widetilde{\delta[\mathcal{D}]}+\bar{\nabla}_{\mathcal{C}}(\alpha)
$$

where $\alpha$ is a section of $\mathcal{H}_{\mathcal{C}}^{1,0}$, then we get

$$
\begin{aligned}
w\left(<(\widetilde{\delta[\mathcal{D}]})^{\prime}, \tilde{\gamma}_{1}>\right) & =w\left(<\widetilde{\delta[\mathcal{D}]}, \tilde{\gamma}_{1}>\right)+w\left(<\bar{\nabla}_{\mathcal{C}}(\alpha), \tilde{\gamma}_{1}>\right) \\
& =w\left(<\widetilde{\delta[\mathcal{D}]}, \tilde{\gamma}_{1}>\right)-w\left(<\alpha, \bar{\nabla}_{\mathcal{C}}\left(\tilde{\gamma}_{1}\right)>\right) .
\end{aligned}
$$

Furthermore, the class $\bar{\nabla}_{\mathcal{C}}\left(\tilde{\gamma}_{1}\right)$, hence also the contraction $-w\left(<\alpha, \bar{\nabla}_{\mathcal{C}}\left(\tilde{\gamma}_{1}\right)>\right)$, is $\bar{\nabla}_{\mathcal{Y}}$-exact for the following reason. Recall that $\tilde{\gamma}_{1}$ is the projection of a $\left.\bar{\nabla}_{\mathcal{C} \times_{B}} \mathcal{Y}_{\text {-closed section } \tilde{\gamma} \text { of }\left(R^{n} \pi_{*}^{\prime} \Omega_{\mathcal{C} \times_{B}}^{n} \mathcal{Y} / B\right.}\right)_{s}$. This section has a Künneth decomposition $\tilde{\gamma}=\tilde{\gamma}_{1}+\tilde{\gamma}_{2}+\ldots$ with $\tilde{\gamma}_{2} \in \Gamma\left(U, \Omega_{U}^{s-1} \otimes \mathcal{H}_{\mathcal{C}}^{0,1} \otimes\right.$ $\left.\mathcal{H}_{\mathcal{Y}}^{n-s+1, n-1}\right)$. The condition $\bar{\nabla}_{\mathcal{C} \times_{U} \mathcal{Y}_{U}}(\tilde{\gamma})=0$ then implies that

$$
\bar{\nabla}_{\mathcal{C}}\left(\tilde{\gamma}_{1}\right)=-\bar{\nabla}_{\mathcal{Y}}\left(\tilde{\gamma}_{2}\right)
$$

Proof of Lemma 2.10. We apply formula (2.18). We observe that the map

$$
p_{2 *}: R^{n+1} \pi_{*}^{\prime} \Omega_{\mathcal{C} \times_{U} \mathcal{Y}_{U}}^{n+1} \rightarrow R^{n} \pi_{*} \Omega_{\mathcal{Y}_{U}}^{n}
$$




\section{Claire Voisin}

is obtained by composing the maps $I$ and $g$, where

$$
g: R^{n+1} \pi_{*}^{\prime} \Omega_{\mathcal{C} \times_{U} \mathcal{Y}_{U}}^{n+1} \rightarrow R^{n+1} \pi_{*}^{\prime}\left(p_{1}^{*} \Omega_{\mathcal{C} / U} \otimes p_{2}^{*} \Omega_{\mathcal{Y}_{U}}^{n}\right)
$$

is induced by the morphism

$$
\Omega_{\mathcal{C} \times_{U} \mathcal{Y}_{U}}^{n+1} \rightarrow p_{1}^{*} \Omega_{\mathcal{C} / U} \otimes p_{2}^{*} \Omega_{\mathcal{Y}_{U}}^{n}
$$

coming from the exact sequence

$$
0 \rightarrow p_{2}^{*} \Omega_{\mathcal{Y}_{U}} \rightarrow \Omega_{\mathcal{C} \times_{U} \mathcal{Y}_{U}} \rightarrow p_{1}^{*} \Omega_{\mathcal{C} / U} \rightarrow 0
$$

and

$$
I: R^{n+1} \pi_{*}^{\prime}\left(p_{1}^{*} \Omega_{\mathcal{C} / U} \otimes p_{2}^{*} \Omega_{\mathcal{Y}_{U}}^{n}\right) \rightarrow R^{n} \pi_{*} \Omega_{\mathcal{Y}_{U}}^{n}
$$

is the integration over the fiber of $p_{2}$, which can also be obtained by writing $\pi^{\prime}=\pi \circ p_{2}$ and sending $R^{n+1} \pi_{*}^{\prime}\left(p_{1}^{*} \Omega_{\mathcal{C} / U} \otimes p_{2}^{*} \Omega_{\mathcal{Y}_{U}}^{n}\right)$ to

$$
R^{n} \pi_{*}\left(R^{1} p_{2 *}\left(p_{1}^{*} \Omega_{\mathcal{C} / U} \otimes p_{2}^{*} \Omega_{\mathcal{Y}_{U}}^{n}\right)\right)=R^{n} \pi_{*}\left(\Omega_{\mathcal{Y}_{U}}^{n}\right)
$$

by the corresponding Leray spectral sequence.

We apply the morphism $g$ to the class $p_{1}^{*}[\mathcal{D}]^{1,1} \cdot\left[\Gamma_{s}\right]^{n, n}$. As $[\mathcal{D}]^{1,1} \in H^{1}\left(\mathcal{C}, \Omega_{\mathcal{C}}\right)$ vanishes in $H^{0}\left(U, R^{1} \rho_{*} \Omega_{\mathcal{C} / U}\right)$, we find that

$$
g\left(p_{1}^{*}[\mathcal{D}]^{1,1} \cdot\left[\Gamma_{s}\right]^{n, n}\right)=\widetilde{\delta[\mathcal{D}]} \cdot g(\gamma) \text { in } \Gamma\left(U, R^{n+1} \pi_{*}^{\prime}\left(p_{1}^{*} \Omega_{\mathcal{C} / U} \otimes p_{2}^{*} \Omega_{\mathcal{Y}_{U}}^{n}\right)\right),
$$

where $g(\gamma)$ is the image of $\gamma \in \Gamma\left(U, R^{n} \pi_{*}^{\prime} \Omega_{\mathcal{C} \times_{U} \mathcal{Y}_{U}}^{n}\right)$ in $\Gamma\left(U, R^{n} \pi_{*}^{\prime}\left(p_{1}^{*} \Omega_{\mathcal{C} / U} \otimes p_{2}^{*} \Omega_{\mathcal{Y}_{U}}^{n-1}\right)\right)$ and $\widetilde{\delta[\mathcal{D}]}$ is any lift of $[\mathcal{D}]$ in $H^{1}\left(\mathcal{C} \rho^{*} \Omega_{U}\right)=\Gamma\left(U, \mathcal{H}_{\mathcal{C}}^{0,1} \otimes \Omega_{U}\right)$.

We now observe that for $g(\gamma) \in \Gamma\left(U, R^{n} \pi_{*}^{\prime}\left(p_{1}^{*} \Omega_{\mathcal{C} / U} \otimes p_{2}^{*} L^{s-1} \Omega_{\mathcal{Y}_{U}}^{n-1}\right)\right.$, and $\widetilde{\delta[\mathcal{D}]} \in H^{1}\left(\mathcal{C}, \rho^{*} \Omega_{U}\right)$, we have

$$
I(\widetilde{\delta[\mathcal{D}}] \cdot g(\gamma))=w\left(<\delta[\mathcal{D}], \gamma_{1}>\right) \text { in } \Gamma\left(U, R^{n} \pi_{*} \Omega_{\mathcal{Y}_{U}}^{n} / L^{s+1} R^{n} \pi_{*} \Omega_{\mathcal{Y}_{U}}^{n}\right) .
$$

To prove $(2.24)$, we choose a class $\mu \in H^{1}\left(\Omega_{\mathcal{C} / U}\right)$ which does not vanish in $H^{0}\left(U, R^{1} \rho_{*} \Omega_{\mathcal{C} / U}\right)$ (for example the first Chern class of a relatively ample line bundle on $\mathcal{C}$ ). The class $g(\gamma) \in \Gamma\left(U, R^{n} \pi_{*}^{\prime}\left(p_{1}^{*} \Omega_{\mathcal{C} / U} \otimes p_{2}^{*} L^{s-1} \Omega_{\mathcal{Y}_{U}}^{n-1}\right)\right)$ can be written as

$$
g(\gamma)=p_{1}^{*} \mu \cdot p_{2}^{*} \eta+p_{2}^{*} \gamma_{1},
$$

with $\eta \in \Gamma\left(U, R^{n-1} \pi_{*}\left(L^{s-1} \Omega_{\mathcal{Y}_{U}}^{n-1}\right)\right)$ and $\gamma_{1} \in \Gamma\left(U, \mathcal{H}_{\mathcal{C}}^{1,0} \otimes R^{n} \pi_{*}\left(L^{s-1} \Omega_{\mathcal{Y}_{U}}^{n-1}\right)\right)$. We then find that

$$
I\left(\widetilde{\delta[\mathcal{D}]} \cdot p_{1}^{*} \mu \cdot p_{2}^{*} \eta\right)=\rho_{*}(\widetilde{\delta[\mathcal{D}]} \cdot \mu) \cdot \eta
$$

which is 0 since we assumed $U$ affine, so $\widetilde{\delta[\mathcal{D}]} \cdot \mu=0$ in $H^{2}\left(\mathcal{C}, \Omega_{\mathcal{C} / U}\right)$. On the other hand, it is clear that for

$$
\widetilde{\delta[\mathcal{D}]} \in \Gamma\left(U, \Omega_{U}\right) \otimes H^{1}\left(\mathcal{O}_{\mathcal{C}}\right)=\Gamma\left(U, \Omega_{U} \otimes \mathcal{H}_{\mathcal{C}}^{0,1}\right)
$$

we have

$$
I\left(\widetilde{\delta[\mathcal{D}]} \cdot p_{2}^{*} \gamma_{1}\right)=w\left(<\widetilde{\delta[\mathcal{D}]}, \gamma_{1}>\right)
$$

because for any $u \in H^{1}\left(\mathcal{C}, \mathcal{O}_{\mathcal{C}}\right)=\Gamma\left(U, R^{1} \rho_{*} \mathcal{O}_{\mathcal{C}}\right)$ and $\omega \in \Gamma\left(U, \mathcal{H}_{\mathcal{C}}^{1,0}\right)$ with cup product $u \cdot \omega \in$ $H^{1}\left(\mathcal{C}, \Omega_{\mathcal{C} / U}\right)$, we have $I(u \cdot \omega)=<u, \omega>$.

Combining (2.23) and (2.24) clearly proves the desired formula (2.22).

We now turn to a general family $\mathcal{Y} \rightarrow B$. The proof in fact works in exactly the same way, except that we have to replace the natural splitting of the Leray filtration on $H^{n}\left(Y_{t}, \Omega_{\mathcal{Y} \mid Y_{t}}^{n}\right)$ given 


\section{INFINITESIMAL INVARIANTS AND 1-CYCLES IN JACOBIANS}

by the character decomposition by another one, which is not canonical and depends only on the choice of a polarization on the family $\mathcal{Y} \rightarrow B$, that is, a line bundle $\mathcal{L}$ on $\mathcal{Y}$ which is ample on the fibers $\mathcal{Y}_{t}$.

Proposition 2.11. Assume that the base $B$ is smooth quasi-projective. Given a relative polarization $\mathcal{L}$ on $\mathcal{Y} \stackrel{\pi}{\rightarrow} B$, there is a canonically induced splitting of the filtration on $H^{q}\left(\mathcal{Y}_{t}, \Omega_{\mathcal{Y}_{\mid \mathcal{Y}_{t}}^{p}}\right)$ or of the sheaves $R^{q} \pi_{*} \Omega_{\mathcal{Y}}^{p}$ induced by the filtration $L$ on $\Omega_{\mathcal{Y}_{\mid \mathcal{Y}_{t}}^{p}}$. This splitting is functorial with respect to pull-back maps.

The proof of Proposition 2.11 is given below. Choosing the pulled-back line bundle $p_{2}^{*} \mathcal{L}$ on the pulled-back family $\mathcal{C} \times_{U} \mathcal{Y}$, we get a similar decomposition of $H^{n}\left(\mathcal{C}_{t} \times \mathcal{Y}_{t}, \Omega_{\mathcal{C} \times_{U}}^{n} \mathcal{Y}_{\mid \mathcal{C}_{t} \times \mathcal{Y}_{t}}\right)$ which splits the filtration $L$ on this space, relative to the projection $\mathcal{C} \times_{U} \mathcal{Y} \rightarrow \mathcal{C}$.

The decompositions are compatible with pull-back and push-forward maps, and the rest of the argument works the same way as before. This concludes the proof of Proposition 2.4.

Proof of Proposition 2.11. We could do it by hand, but it is quicker to see this as a consequence of Deligne's canonical splitting result in [Del91]. Deligne proves that the choice of a relative polarization on $\pi: \mathcal{Y} \rightarrow B$ induces a canonical quasi-isomorphism

$$
R \pi_{*} \mathbb{Q} \cong \oplus_{i} R^{i} \pi_{*} \mathbb{Q} .
$$

It follows that there are canonical cohomology classes $\tilde{\pi}_{k} \in H^{2 d}\left(\mathcal{Y} \times_{B} \mathcal{Y}, \mathbb{Q}\right), d=\operatorname{dim} \mathcal{Y}_{t}$, whose images in $H^{0}\left(B, R^{2 d}(\pi, \pi)_{*} \mathbb{Q}\right)$ are the Künneth projectors $\pi_{k}$ :

$$
\begin{aligned}
R^{2 d}(\pi, \pi)_{*} \mathbb{Q} & =\oplus_{i+j=2 d} R^{i} \pi_{*} \mathbb{Q} \otimes R^{j} \pi_{*} \mathbb{Q} \\
& =\oplus_{j} H o m\left(R^{j} \pi_{*} \mathbb{Q}, R^{j} \pi_{*} \mathbb{Q}\right) \ni \pi_{j}:=I d_{R^{j} \pi_{*} \mathbb{Q}} .
\end{aligned}
$$

From the construction of [Del91], it is clear that these classes $\tilde{\pi}_{k} \in H^{2 d}\left(\mathcal{Y} \times{ }_{B} \mathcal{Y}, \mathbb{Q}\right)$ are Hodge classes, which means that they extend to any smooth compactification of $\mathcal{Y} \times{ }_{B} \mathcal{Y}$ and are Hodge classes there. It then follows that these classes have Dolbeault counterparts:

$$
\tilde{\pi}_{k}^{d, d} \in H^{d}\left(\mathcal{Y} \times_{B} \mathcal{Y}, \Omega_{\mathcal{Y} \times_{B}}^{d} \mathcal{Y}\right) .
$$

We can now use the $\tilde{\pi}_{k}^{d, d}$ as acting on the relative Dolbeault cohomology of $\mathcal{Y}$. Namely, denoting by $p r_{1}$ and $p r_{2}$ the projections from $\mathcal{Y} \times_{B} \mathcal{Y}$ to $B$, we have maps:

$$
p r_{2 *} \circ\left(\tilde{\pi}_{k}\right) \cup \circ p r_{1}^{*}: R^{q} \pi_{*} \Omega_{\mathcal{Y}}^{p} \rightarrow R^{q} \pi_{*} \Omega_{\mathcal{Y}}^{p} .
$$

They similarly act on the fibers at $t$ :

$$
\operatorname{pr}_{2 *} \circ\left(\tilde{\pi}_{k}\right) \cup \circ \operatorname{pr}_{1}^{*}: H^{q}\left(\mathcal{Y}_{t}, \Omega_{\mathcal{Y} \mid \mathcal{Y}_{t}}^{p}\right) \rightarrow H^{q}\left(\mathcal{Y}_{t}, \Omega_{\mathcal{Y} \mid \mathcal{Y}_{t}}^{p}\right)
$$

These maps are compatible with the $L$-filtration and induce a morphism of spectral sequences associated to the filtration $L$. On the other hand, their action on $E_{1}^{s, q-s}=\Omega_{B, t}^{s} \otimes H^{q-s}\left(\mathcal{Y}_{t}, \Omega_{\mathcal{Y}_{\mid \mathcal{Y}_{t}}^{p}}\right)$ is induced by $\pi_{k}$, hence it is equal to 0 for $p+q-s \neq k$ and the identity if $p+q-s=k$, and similarly for $E_{2}^{s, q-s}=E_{\infty}^{s, q-s}$. It follows that we have induced maps

$$
p r_{2 *} \circ\left(\tilde{\pi}_{p+q-s}\right) \cup \circ p r_{1}^{*}: L^{s} H^{q}\left(\mathcal{Y}_{t}, \Omega_{\mathcal{Y}_{\mid \mathcal{Y}_{t}}^{p}}\right) \rightarrow L^{s} H^{q}\left(\mathcal{Y}_{t}, \Omega_{\mathcal{Y}_{\mid \mathcal{Y}_{t}}^{p}}\right)
$$

which have the property that after passing to the graded vector bundle $G r_{L}$, they induce the zero map on $E_{2}^{s^{\prime}, q-s^{\prime}}$ for $s^{\prime}>s$ and the identity map on $E_{2}^{s, q-s}=E_{\infty}^{s, q-s}$. But then a sufficiently high power of these maps vanishes on $L^{s+1} H^{q}\left(\mathcal{Y}_{t}, \Omega_{\mathcal{Y}_{\mid \mathcal{Y}_{t}}^{p}}\right)$ and thus factors through $E_{\infty}^{s, q-s}$ to give 


\section{Claire Voisin}

a retraction

hence a canonical splitting

$$
E_{\infty}^{s, q-s} \rightarrow L^{s} H^{q}\left(\mathcal{Y}_{t}, \Omega_{\mathcal{Y}_{\mid \mathcal{Y}_{t}}^{p}}\right)
$$

$$
L^{s} H^{q}\left(\mathcal{Y}_{t}, \Omega_{\mathcal{Y} \mid \mathcal{Y}_{t}}^{p}\right)=L^{s+1} H^{q}\left(\mathcal{Y}_{t}, \Omega_{\mathcal{Y}_{\mid \mathcal{Y}_{t}}^{p}}\right) \oplus E_{\infty}^{s, q-s}, E_{\infty}^{s, q-s}=E_{2}^{s, q-s}
$$

\section{Codimension $g-1$ cycles modulo algebraic equivalence in Jacobians}

\subsection{Constructing test elements in the dual space}

We now consider a family $\mathcal{C} \rightarrow B$ of curves of genus $g$ and the associated Jacobian fibration $\pi: \mathcal{J} \rightarrow B$. We choose an embedding $\mathcal{C} \subset \mathcal{J}$, which provides a codimension $g-1$ cycle $\mathcal{Z} \in \mathrm{CH}^{g-1}(\mathcal{J}) /$ alg and we want to study the cycles $\mathcal{Z}_{s} \in \mathrm{CH}^{g-1}(\mathcal{J})_{s} /$ alg. Our goal is to exhibit such families with a nontrivial infinitesimal invariant

$$
\delta\left[\mathcal{Z}_{s}\right]_{a l g} \in I_{s, g-1-s, g-1}:=\Gamma\left(B, \frac{\operatorname{Ker}\left(\bar{\nabla}_{s, g-1-s, g-1}\right)}{\Omega_{B} \wedge \operatorname{Ker}\left(\bar{\nabla}_{s-1, g-s-1, g-1}\right)}\right)
$$

for $s \geqslant 2$. Here $\bar{\nabla}$ is the $\bar{\nabla}$-map for the Hodge bundles of the family $\mathcal{J} \rightarrow B$. The infinitesimal variation of Hodge structure on the cohomology of the fibers of the family $\left(\mathcal{J}_{t}\right)_{t \in B}$ of Jacobians is deduced from the one of the family of curves $\left(\mathcal{C}_{t}\right)_{t \in B}$, in the obvious way. Namely, it coincides with the latter in degree 1 and with its $k$-th exterior power for higher $k$.

The map

$$
\bar{\nabla}_{s, p, q}: \Omega_{B}^{s} \otimes \mathcal{H}^{p, q} \rightarrow \Omega_{B}^{s+1} \otimes \mathcal{H}^{p-1, q+1}
$$

has for transposed map

$$
{ }^{t} \bar{\nabla}_{s+1, g-p+1, g-q-1}: \bigwedge^{s+1} T_{B} \otimes \mathcal{H}^{g-p+1, g-q-1} \rightarrow \bigwedge^{s} T_{B} \otimes \mathcal{H}^{g-p, g-q} .
$$

This map is given by the formula

$$
\begin{gathered}
{ }^{t} \bar{\nabla}_{s+1, g-p+1, g-q-1}\left(u_{1} \wedge \ldots \wedge u_{s+1} \otimes \omega_{1} \wedge \ldots \wedge \omega_{g-p+1} \otimes \eta_{1} \wedge \ldots \wedge \eta_{g-q-1}\right) \\
=\sum_{1 \leqslant i \leqslant s+1,1 \leqslant j \leqslant g-p+1}(-1)^{i+j} u_{1} \wedge \ldots \wedge \hat{u}_{i} \wedge \ldots \wedge u_{s+1} \otimes \omega_{1} \wedge \ldots \wedge \hat{\omega}_{j} \wedge \ldots \wedge \omega_{g-p+1} \\
\otimes{ }^{t} \bar{\nabla}_{1,1,0}\left(u_{i} \otimes \omega_{j}\right) \wedge \eta_{1} \wedge \ldots \wedge \eta_{g-q-1} .
\end{gathered}
$$

Fix a point $b \in B$. The dual of the space of infinitesimal invariants

$$
\frac{\operatorname{Ker}\left(\bar{\nabla}_{s, g-1-s, g-1, b}: \Omega_{B, b}^{s} \otimes \mathcal{H}_{b}^{g-1-s, g-1} \rightarrow \Omega_{B, b}^{s+1} \otimes \mathcal{H}_{b}^{g-2-s, g}\right)}{\Omega_{B, b} \wedge \operatorname{Ker}\left(\bar{\nabla}_{s-1, g-s-1, g-1, b}: \Omega_{B, b}^{s-1} \otimes \mathcal{H}_{b}^{g-1-s, g-1} \rightarrow \Omega_{B, b}^{s} \otimes \mathcal{H}_{b}^{g-2-s, g}\right)}
$$

is the quotient of the space

$$
I_{s, g-1-s, g-1}^{*}:=\operatorname{Ker}\left(\bigwedge^{s} T_{B, b} \otimes \mathcal{H}_{b}^{s+1,1} \rightarrow T_{B, b} \otimes \frac{\bigwedge^{s-1} T_{B, b} \otimes \mathcal{H}_{b}^{s+1,1}}{\operatorname{Im}^{t} \bar{\nabla}_{s, s+2,0, b}}\right)
$$

by the subspace

$$
\operatorname{Im}\left({ }^{t} \bar{\nabla}_{s+1, s+2,0, b}: \bigwedge^{s+1} T_{B, b} \otimes \mathcal{H}_{b}^{s+2,0} \rightarrow \bigwedge^{s} T_{B, b} \otimes \mathcal{H}_{b}^{s+1,1}\right)
$$




\section{INFINITESIMAL INVARIANTS AND 1-CYCLES IN JACOBIANS}

where $\mathcal{H}_{b}^{p, q}=\bigwedge^{p} H^{0}\left(C, K_{C}\right) \otimes \bigwedge^{q} H^{1}\left(C, \mathcal{O}_{C}\right)$. The following lemma describes the simplest possible elements in the space $I_{s, g-1-s, g-1}^{*}$.

Lemma 3.1. Assume that we have

(i) an $s$-dimensional subspace $W=<u_{1}, \ldots, u_{s}>\subset T_{B, b}$,

(ii) an $s+1$-dimensional subspace $K=<\omega_{1}, \ldots, \omega_{s+1}>\subset \mathcal{H}_{b}^{1,0}$ and an $s$-dimensional subspace $K_{1}=<\mu_{1}, \ldots, \mu_{s}>\subset \mathcal{H}_{b}^{1,0}$,

(iii) an element $\eta \in \mathcal{H}_{b}^{0,1}$,

such that

(i) For any $i \in\{1, \ldots, s\}$ and $j \in\{1, \ldots, s+1\},{ }^{t} \bar{\nabla}\left(u_{i} \otimes \omega_{j}\right)=0$ in $\mathcal{H}_{b}^{0,1}$.

(ii) For any $i \in\{1, \ldots, s\}$ and $j \in\{1, \ldots, s\},{ }^{t} \bar{\nabla}\left(u_{i} \otimes \mu_{j}\right)=\lambda_{i j} \eta$ in $\mathcal{H}_{b}^{0,1}$, where the $(s, s)$-matrix $\left(\lambda_{i j}\right)$ is invertible.

Then $w:=u_{1} \wedge \ldots \wedge u_{s} \otimes \omega_{1} \wedge \ldots \wedge \omega_{s+1} \otimes \eta$ belongs to the subspace $I_{s, g-1-s, g-1}^{*}$ of (3.25).

Proof. Indeed, we may assume that $\lambda_{i j}=\delta_{i j}$ after changing the basis $u_{i}$ if necessary. Then the image of $u_{1} \wedge \ldots \wedge u_{s} \otimes \omega_{1} \wedge \ldots \wedge \omega_{s+1} \otimes \eta$ in $T_{B, b} \otimes \wedge^{s-1} T_{B, b} \otimes \mathcal{H}^{s+1,1}$ is equal to $\sum_{i}(-1)^{i} u_{i} \otimes$ $u_{1} \wedge \ldots \wedge \hat{u}_{i} \wedge \ldots \wedge u_{s} \otimes \omega_{1} \wedge \ldots \wedge \omega_{s+1} \otimes \eta$. On the other hand, for any $i$, we have:

$$
\begin{aligned}
& u_{1} \wedge \ldots \wedge \hat{u}_{i} \wedge \ldots \wedge u_{s} \otimes \omega_{1} \wedge \ldots \wedge \omega_{s+1} \otimes \eta \\
& =(-1)^{i t} \bar{\nabla}_{s, s+2,0, b}\left(u_{1} \wedge \ldots \wedge u_{s} \otimes \mu_{i} \wedge \omega_{1} \wedge \ldots \wedge \omega_{s+1}\right) .
\end{aligned}
$$

Thus $u_{1} \wedge \ldots \wedge u_{s} \otimes \omega_{1} \wedge \ldots \wedge \omega_{s+1} \otimes \eta \in \operatorname{Ker}\left(\bigwedge^{s} T_{B, b} \otimes \mathcal{H}_{b}^{s+1,1} \rightarrow T_{B, b} \otimes \frac{\bigwedge^{s-1} T_{B, b} \otimes \mathcal{H}_{b}^{s+1,1}}{\operatorname{Im}^{t} \bar{\nabla}_{s, s+2,0, b}}\right)$.

\subsection{The Ikeda family}

The Ikeda family [Ike03] is simply the family of plane curves which are cyclic covers of $\mathbb{P}^{1}$ of degree $d$ ramified along a degree $d$ divisor. In other words, the general equation $f$ of such a plane curve takes the form

$$
f\left(Y, X_{0}, X_{1}\right)=Y^{d}-f_{0}\left(X_{0}, X_{1}\right)
$$

(in homogeneous coordinates $Y, X_{0}, X_{1}$ on $\mathbb{P}^{2}$ ) for some homogeneous polynomial $f_{0}$ of degree $d$. This family is parameterized by the quotient $U / P G l(2)$ where $U \subset \mathbb{P}\left(H^{0}\left(\mathbb{P}^{1}, \mathcal{O}_{\mathbb{P}^{1}}(d)\right)\right)$ is the open set parameterizing reduced divisors, hence smooth plane curves.

The tangent space to this quotient at a general point $f_{0}$ is the quotient

$$
R_{f_{0}}^{d}=S_{X}^{d} / J_{f_{0}}^{d}
$$

where $S_{X}^{k}:=H^{0}\left(\mathbb{P}^{1}, \mathcal{O}_{\mathbb{P}^{1}}(k)\right)$ and $J_{f_{0}}^{k} \subset S^{k}$ is the degree $k$ part of the Jacobian ideal of $f_{0}$, generated by the partial derivatives $\partial f_{0} / \partial X_{i}$.

We consider the universal family $\mathcal{C} \rightarrow B$, where $B \subset U$ is any slice for the action of $P G l(2)$ and the corresponding infinitesimal variation of Hodge structure at a point $b \in B$ corresponding to an equation $f$ as above. Let us exhibit, for any such $f$, data as in Lemma 3.1 with $s=d-3$.

We recall from [CG80] (see also [Voi03, 6.2.1]) that the infinitesimal variation of Hodge structure of a hypersurface defined by a homogeneous equation $f$ is governed by the product in the Jacobian ring $R_{f}$ of $f$, quotient of the polynomial ring by the ideal generated by the partial derivatives of $f$. In the case of a curve $C$ of degree $d$ in $\mathbb{P}^{2}$ defined by an equation $f$, we get

$$
H^{1,0}(C) \cong R_{f}^{d-3}, H^{0,1}(C) \cong R_{f}^{2 d-3}
$$




\section{Claire Voisin}

and the infinitesimal variation of Hodge structure restricted to the family of plane deformations of $C$ identifies

$$
{ }^{t} \bar{\nabla}_{1,1,0}: T_{B, b} \otimes H^{1,0}(C) \rightarrow H^{0,1}(C)
$$

with the multiplication map

$$
R_{f}^{d} \otimes R_{f}^{d-3} \rightarrow R_{f}^{2 d-3},
$$

where $B$ is a slice for the $\operatorname{PGl}(3)$-action and $b \in B$ is the parameter for $C$.

Let now $f=Y^{d}-f_{0}\left(X_{0}, X_{1}\right)$, which is homogeneous of degree $d$ in the three variables $Y, X_{0}, X_{1}$. Note that $\partial f / \partial X_{i}=-\partial f_{0} / \partial X_{i}$ for $i=0,1$, so that $R_{f}$ is a module over $R_{f_{0}}$.

Consider the following subspaces:

$$
W:=R_{f_{0}}^{d} \subset R_{f}^{d}, K:=R_{f_{0}}^{d-3}=S_{X}^{d-3} \text { and } K_{1}:=Y R_{f_{0}}^{d-4}=Y S_{X}^{d-4} .
$$

Note that $\operatorname{dim} W=d-3, \operatorname{dim} K=d-2$ and $\operatorname{dim} K_{1}=d-3$. We have the following result.

Lemma 3.2. (i) For any $u \in W, \omega \in K$, we have ${ }^{t} \bar{\nabla}(u \otimes \omega)=0$.

(ii) There is an element $\eta \in H^{0,1}(C)$ such that the map $W \otimes K_{1} \rightarrow H^{0,1}(C), u \otimes \omega \mapsto{ }^{t} \bar{\nabla}(u \otimes \omega)$ takes the form

$$
{ }^{t} \bar{\nabla}(u \otimes \omega)=\lambda(u, \omega) \eta
$$

where the bilinear form $\lambda$ gives a perfect pairing between $W$ and $K_{1}$.

Proof. (i) This follows from the fact that $R_{f_{0}}^{2 d-3}=0$.

(ii) We choose for $\eta$ the generator of the 1-dimensional vector space $Y R_{f_{0}}^{2 d-4}$, which means geometrically (cf. [CG80]) that

$$
\eta=\operatorname{Res}_{C} \frac{P\left(X_{0}, X_{1}\right) Y \Omega}{f^{2}} \text { in } H^{0,1}(C),
$$

with $\Omega=X_{0} d X_{1} \wedge d Y-X_{1} d X_{0} \wedge d Y+Y d X_{0} \wedge d X_{1} \in H^{0}\left(\mathbb{P}^{2}, K_{\mathbb{P}^{2}}(3)\right)$ and $P$ generic of degree $2 d-4$ in the variables $X_{0}, X_{1}$.

Then statement (ii) follows from Macaulay's theorem (cf. [Voi03, 6.2.2]), which says that $R_{f_{0}}^{2 d-4}=\mathbb{C}$ and that the multiplication map $R_{f_{0}}^{d} \otimes R_{f_{0}}^{d-4} \rightarrow R_{f_{0}}^{2 d-4}$ is a perfect pairing.

We now get the following corollary. Here we consider the Ikeda family $\mathcal{C} \rightarrow B$ (defined over a slice of the quotient map $\left.\mathbb{P}\left(S_{X}^{d}\right) \rightarrow \mathbb{P}\left(S_{X}^{d}\right) / P G l(2)\right)$, the associated Jacobian fibration $\pi: \mathcal{J} \rightarrow B$, an embedding $\mathcal{C} \rightarrow \mathcal{J}$ giving rise to a codimension $g-1$ cycle $\mathcal{Z}$ of $\mathcal{J}$ and its (relative) Beauville component $\mathcal{Z}_{d-3}$.

Corollary 3.3. Choose a basis $u_{1}, \ldots, u_{d-3}$ of $W$ and a basis $\omega_{1}, \ldots, \omega_{d-2}$ of $K$. Let $\eta$ be a generator of $Y R_{f_{0}}^{2 d-4}=\mathbb{C} \subset H^{1}\left(C, \mathcal{O}_{C}\right)$, where the curve $C$ is defined by $Y^{d}=f_{0}\left(X_{0}, X_{1}\right)$. Then

$$
w:=u_{1} \wedge \ldots \wedge u_{d-3} \otimes \omega_{1} \wedge \ldots \wedge \omega_{d-2} \otimes \eta
$$

belongs to the space $I_{d-3, g-d+2, g-1}^{*}$, where $g=\frac{1}{2} d(d-3)+1$.

Proof. We only have to observe that the space $W \subset H^{1}\left(C, T_{C}\right)$ is exactly the tangent space to the Ikeda family, so that $w$ belongs to $\bigwedge^{d-3} T_{B, b} \otimes H^{d-2,1}(J C)$. Applying Lemmas 3.1 and 3.2, we also get that the image of $w$ in $T_{B, b} \otimes \bigwedge^{d-4} T_{B, b} \otimes H^{d-2,1}(J C)$ vanishes in the quotient

$$
T_{B, b} \otimes \frac{\bigwedge^{d-4} T_{B, b} \otimes H^{d-2,1}(J C)}{\operatorname{Im}^{t} \bar{\nabla}_{d-3, d-1,0}},
$$




\section{INFINITESIMAL INVARIANTS AND 1-CYCLES IN JACOBIANS}

which proves the result.

\subsection{End of the proof of Theorem 1.6}

This section is devoted to the proof of the following result.

Proposition 3.4. Let $f_{0} \in S_{X}^{d}$ define a smooth divisor in $\mathbb{P}^{1}$. Let $C$ be the curve defined by $Y^{d}-f_{0}\left(X_{0}, X_{1}\right)$ and let $w \in I_{d-3, g-d+2, g-1}^{*}$ be the element constructed in Corollary 3.3. Then the pairing

$$
<\delta\left[\mathcal{Z}_{d-3}\right]_{b, a l g}, w>
$$

is nonzero, where $b \in B$ is the point of the Ikeda family $\mathcal{C} \rightarrow B$ parameterizing $C$.

Note that Proposition 3.4 implies Theorem 1.6. Indeed, it implies that for the Ikeda family $\mathcal{C} \rightarrow B$, the infinitesimal invariant $\delta\left[\mathcal{Z}_{d-3}\right]_{b, a l g}$ of the corresponding codimension $g-1$ cycle $\mathcal{Z}$ of $\mathcal{J} \rightarrow B$ is nonzero at the general point of $B$, which implies by Theorem 1.1 that for the very general curve $C$ parameterized by $B$ (hence for the very general plane curve), the Beauville component $Z_{d-3}$ is nonzero in $\mathrm{CH}_{1}(J C)_{d-3} /$ alg.

The proof of Proposition 3.4 will use the following description of the pairing $<\delta\left[\mathcal{Z}_{d-3}\right]_{b, a l g}, w>$. Let $\mathcal{C} \subset \mathcal{J} \rightarrow B$ be a family of curves of genus $g$ and let $w \in I_{s, g-1-s, g-1}^{*}$ be an element constructed as in Lemma 3.1. Assume for simplicity that $\operatorname{dim} B=s$, so that $W=T_{B, b}$, where the point $b$ parameterizes the curve $C:=\mathcal{C}_{b}$. Then the pairing

$$
<\delta\left[\mathcal{Z}_{s}\right]_{b, a l g}, w>
$$

is computed as follows. Since all the forms $\omega_{j} \in H^{0}\left(C, K_{C}\right)$ satisfy ${ }^{t} \bar{\nabla}\left(u_{i} \otimes \omega_{j}\right)=0$ in $H^{1}\left(C, \mathcal{O}_{C}\right)$, they lift to sections of

$$
H^{0}\left(C, \Omega_{\mathcal{C} \mid C}\right)=H^{0}\left(C, \Omega_{\mathcal{J} \mid C}\right)=H^{0}\left(J, \Omega_{\mathcal{J} \mid J}\right),
$$

where $J=J C=\mathcal{J}_{b}$. For any $k$, the multiplication $\mu_{k}$ induces an endomorphism $\mu_{k}^{*}$ of $H^{0}\left(J, \Omega_{\mathcal{J} \mid J}\right)$ which is compatible with the restriction map

$$
H^{0}\left(J, \Omega_{\mathcal{J} \mid J}\right) \rightarrow H^{0}\left(J, \Omega_{J}\right),
$$

the action of $\mu_{k}^{*}$ on the right hand term being the homothety of factor $k$. It follows that there is an unique lift $\tilde{\omega}_{j}$ of $\omega_{j}$ in $H^{0}\left(J, \Omega_{\mathcal{J} \mid J}\right)$ satisfying the property

$$
\mu_{k}^{*}\left(\tilde{\omega}_{j}\right)=k \tilde{\omega}_{j} \text { for any } k .
$$

The $d-2$-form

$$
\tilde{\omega}_{1} \wedge \ldots \wedge \tilde{\omega}_{s+1} \in H^{0}\left(C, \Omega_{\mathcal{J} \mid C}^{s+1}\right)
$$

restricts to a form $\mu \in H^{0}\left(C, \Omega_{\mathcal{C} \mid C}^{s+1}\right)$. There is an isomorphism

$$
\Omega_{\mathcal{C} \mid C}^{s+1} \cong K_{C}
$$

which becomes canonical once $\bigwedge^{s} W$ is trivialized and we trivialize $\bigwedge^{s} W$ using the multivector $u_{1} \wedge \ldots \wedge u_{s}$. We thus obtain from $w$ an element $\mu \cdot \eta \in H^{1}\left(C, K_{C}\right)$.

Lemma 3.5. We have

$$
<\delta\left[\mathcal{Z}_{s}\right]_{b, a l g}, w>=\int_{C} \mu \cdot \eta
$$




\section{Claire Voisin}

Proof. In a general situation $\mathcal{C} \hookrightarrow \mathcal{J} \rightarrow B$, the class $[\mathcal{Z}]_{b}^{g-1, g-1} \in H^{g-1}\left(J C, \Omega_{\mathcal{J} \mid J C}^{g-1}\right)$ is the Dolbeault cohomology class of $\mathcal{C} \subset \mathcal{J}$ and the class $[\mathcal{Z}]_{s, a l g, b}$ is its component lying in the space

$$
H^{g-1}\left(J C, \Omega_{\mathcal{J} \mid J C}^{g-1}\right)_{s}:=\left\{\alpha \in H^{g-1}\left(J C, \Omega_{\mathcal{J} \mid J C}^{g-1}\right), \mu_{k}^{*} \alpha=k^{2 g-2-s}, \forall k\right\} .
$$

For any class $\beta$ in the dual space

$$
H^{1}\left(J C, \Omega_{\mathcal{J} \mid J C}^{N+1}\right), N:=\operatorname{dim} B
$$

we have

$$
<[\mathcal{C}]_{b}^{g-1, g-1}, \beta>=\int_{C} \beta_{\mid \mathcal{C}},
$$

where $\beta_{\mid \mathcal{C}}$ is the image of $\beta$ in $H^{1}\left(C, \Omega_{\mathcal{C} \mid C}^{N+1}\right)$ which is identified with $H^{1}\left(C, K_{C}\right)$ via a trivialization of $\bigwedge^{N} \Omega_{B, b}$. Coming back to our situation above, where $N=s$, the statement thus follows from the following facts:

(i) The choice of a lift $\tilde{\omega}_{j} \in H^{0}\left(\Omega_{\mathcal{J} \mid J C}\right)_{1}$ gives the unique lift

$$
\tilde{w}:=\tilde{\omega}_{1} \wedge \ldots \wedge \tilde{\omega}_{s+1} \otimes \eta \in H^{1}\left(J C, \Omega_{\mathcal{J}^{s+1} \mid J C}\right)_{s+2}
$$

of $w \in G r_{L}^{s+2} H^{1}\left(J C, \Omega_{\mathcal{J} \mid J C}^{s+1}\right)$.

(ii) Writing $[\mathcal{Z}]^{g-1, g-1}=\sum_{i}\left[\mathcal{Z}_{i}\right]^{g-1, g-1}$, we then have

$$
<[\mathcal{Z}]_{b}^{g-1, g-1}, \tilde{w}>=<\left[\mathcal{Z}_{s}\right]_{b}^{g-1, g-1}, \tilde{w}>,
$$

since the coupling is multiplied by $k^{2 g}$ under $\mu_{k}^{*}$, which implies that all the other terms $<\left[\mathcal{Z}_{s^{\prime}}\right]^{g-1, g-1}, \tilde{w}>$ vanish.

The term on the right in (3.30) gives a coupling $<\delta\left[\mathcal{Z}_{s}\right]_{b}^{g-1, g-1}, w>$ which, by the fact that $w$ is in the kernel $I_{d-3, g-d+2, g-1}^{*}$ of (3.25), is equal to the desired coupling $\left\langle\delta\left[\mathcal{Z}_{s}\right]_{b, a l g}^{g-1, g-1}, w>\right.$. On the other hand, the term on the left is computed by formula (3.29). The equality (3.30) thus proves Lemma 3.5.

Proof of Proposition 3.4. We follow the description given in Lemma 3.5. As ${ }^{t} \bar{\nabla}\left(u_{i} \otimes \omega_{j}\right)=0$ for all $i, j$, each $\omega_{j}$ has a canonical lift $\tilde{\omega}_{j} \in H^{0}\left(J, \Omega_{\mathcal{J} \mid J}\right)$. This lift is determined by the condition that $\mu_{k}^{*} \tilde{\omega}_{j}=k \tilde{\omega}_{j}$ for all $k$.

We observe that the cyclic group $G:=\mathbb{Z} / d \mathbb{Z}$ with generator $g$ identified with a $d$-th primitive root of unity $\zeta$ acts over $B$ on the families $\mathcal{C}$ and $\mathcal{J}$, in a compatible way if we embed $\mathcal{C}$ in $\mathcal{J}$ using one of the fixed points of the action of $G$ on the curves. We claim that the forms $\tilde{\omega}_{j}$ satisfy

$$
g^{*} \tilde{\omega}_{j}=\zeta \tilde{\omega}_{j} .
$$

To see this, we write the exact sequence

$$
0 \rightarrow \Omega_{B, b} \otimes \mathcal{O}_{C} \rightarrow \Omega_{\mathcal{C} \mid C} \rightarrow \Omega_{C} \rightarrow 0
$$

which induces the exact sequence

$$
0 \rightarrow \Omega_{B, f} \rightarrow H^{0}\left(C, \Omega_{\mathcal{J} \mid C}\right) \rightarrow K \rightarrow 0
$$

with $K \subset H^{1,0}(C)$ defined as in (3.26). This indeed follows from Macaulay's theorem, which implies that $K=\left\{\alpha \in H^{0}\left(\Omega_{C}\right), \bar{\nabla}(\alpha)=0\right.$ in $\left.\Omega_{B, b} \otimes H^{1}\left(C, \mathcal{O}_{C}\right)\right\}$. The claim then follows from the fact that the $\omega_{j}$, which are the images of $\tilde{\omega}_{j}$ in $H^{0}\left(J, \Omega_{\mathcal{J} / B \mid J}\right)=H^{0}\left(J, \Omega_{J}\right)=H^{1,0}(C)$, satisfy the property

$$
g^{*} \omega_{j}=\zeta \omega_{j}
$$




\section{INFINITESIMAL INVARIANTS AND 1-CYCLES IN JACOBIANS}

and from the fact that the two actions $\mu_{k}^{*}$ and $g^{*}$ on the space

$$
H^{0}\left(J, \Omega_{\mathcal{J} \mid J}\right)=H^{0}\left(C, \Omega_{\mathcal{J} \mid C}\right)=H^{0}\left(C, \Omega_{\mathcal{C} \mid C}\right),
$$

commute, both acting trivially on the space on the left in (3.32) and as a homothety of factor $k$ and $\zeta$, respectively, on the space on the right in (3.32).

As an immediate consequence of (3.31), we find that

$$
g^{*}\left(\tilde{\omega}_{1} \wedge \ldots \wedge \tilde{\omega}_{d-2}\right)=\zeta^{d-2} \tilde{\omega}_{1} \wedge \ldots \wedge \tilde{\omega}_{d-2} \text { in } H^{0}\left(\bigwedge^{d-2} \Omega_{\mathcal{J} \mid C}\right)
$$

hence a fortiori the restricted form $\mu$ which is the image of $\tilde{\omega}_{1} \wedge \ldots \wedge \tilde{\omega}_{d-2} \in H^{0}\left(\bigwedge^{d-2} \Omega\right)_{\mathcal{J} \mid C}$ in $H^{0}\left(\bigwedge^{d-2} \Omega_{\mathcal{C} \mid C}\right)=H^{1,0}(C)$ satisfies

$$
g^{*} \mu=\zeta^{d-2} \mu .
$$

Next we observe that the form $\eta \in H^{0,1}(C)$ defined in (3.27) satisfies

$$
g^{*} \eta=\zeta^{2} \eta
$$

as follows immediately from the formula

$$
\eta=\operatorname{Res}_{C} \frac{P Y \Omega}{f^{2}}
$$

with $g^{*} Y=\zeta Y, g^{*} \Omega=\zeta \Omega$ and $g^{*} f=f, g^{*} P=P$.

The two spaces $H^{1,0}(C)^{\zeta^{d-2}}$ and $H^{0,1}(C)^{\zeta^{2}}$ where $g^{*}$ acts by multiplication by $\zeta^{d-2}$ and $\zeta^{2}$, respectively, are dual and 1-dimensional and the second space is generated by $\eta$. It follows that the pairing $\left\langle\delta\left[\mathcal{Z}_{d-3}\right]_{b, a l g}, w\right\rangle$, which by Lemma 3.5 is computed as the pairing $\langle\mu, \eta\rangle$ between $\mu \in H^{1,0}(C)$ and $\eta \in H^{0,1}(C)$, vanishes if and only if $\mu$ vanishes identically.

It thus suffices to prove that $\mu$ is nonzero. This is done as follows: Recall that the bundle $\Omega_{\mathcal{C} \mid C}$ has rank $d-2$ and possesses the $d-2$ sections $\tilde{\omega}_{j}$. Assume that $\mu$ vanishes in $H^{0}\left(C, \bigwedge^{d-2} \Omega_{\mathcal{C} \mid C}\right)=$ $H^{0}\left(C, K_{C}\right)$. Then these sections generate a subbundle $N \subset \Omega_{\mathcal{C} \mid C}$ of rank $\leqslant d-3$, and it follows that if $x \in C$ is a general point, there is a section $\tilde{\omega}_{x} \in<\tilde{\omega}_{1}, \ldots, \tilde{\omega}_{d-2}>$ vanishing at $x$. As all the sections $\tilde{\omega}_{j}$ satisfy $g^{*} \tilde{\omega}_{j}=\zeta \tilde{\omega}_{j}$, it follows that $\tilde{\omega}_{x}$ also vanishes at $g x, g^{2} x, \ldots, g^{d-1} x$, so that $\tilde{\omega}_{x}$ is in fact a section of $\Omega_{\mathcal{C} \mid C}(-1)$. It is easy to check, however, using the multiplication in the Jacobian ring $R_{f}$, that $H^{0}\left(C, \Omega_{\mathcal{C} \mid C}(-1)\right)=0$. Indeed, we have the exact sequence

$$
0 \rightarrow \Omega_{B, b} \otimes \mathcal{O}_{C}(-1) \rightarrow \Omega_{\mathcal{C} \mid C}(-1) \rightarrow K_{C}(-1) \rightarrow 0
$$

and the induced map

$$
H^{0}\left(C, K_{C}(-1)\right) \rightarrow \Omega_{B, b} \otimes H^{1}\left(C, \mathcal{O}_{C}(-1)\right)=\operatorname{Hom}\left(R_{f_{0}}^{d}, R_{f}^{2 d-4}\right)
$$

is given by multiplication in the Jacobian ring of $f$. Such a section would provide a nonzero element of

$$
R_{f}^{d-4}=\oplus_{i=0}^{i=d-1} Y^{i} R_{f_{0}}^{d-4-i}
$$

which is annihilated by multiplication by all elements of $W=R_{f_{0}}^{d}$, and this does not exist by Macaulay's Theorem.

\section{Comparison with Ikeda's invariants}

As already mentioned in the introduction, Ikeda [Ike03] proves the nonvanishing of the cycle $Z_{d-3}$ for the very general curve in the Ikeda family of plane curves of degree $d$, modulo a certain 


\section{Claire Voisin}

subgroup of $\mathrm{CH}^{g-1}(J C)$. Here the cycle $Z_{d-3}$ is obtained by embedding the curve $C$ in $J C$ using a general point $p \in J C$.

The infinitesimal invariant used by Ikeda is the projection of

$$
\delta\left[\mathcal{Z}_{d-3, b}\right] \in \frac{\operatorname{Ker} \bar{\nabla}_{d-3, g-d+2, g-1, b} \subset \Omega_{B, b}^{d-3} \otimes H^{g-d+2, g-1}(J C)}{\operatorname{Im} \bar{\nabla}_{d-4, g-d+3, g-2}}
$$

modulo the subspace

$$
\Omega_{B, b}^{d-3} \otimes \operatorname{Ker} \bar{\nabla}_{0, g-d+2, g-1, b} \subset \operatorname{Ker} \bar{\nabla}_{d-3, g-d+2, g-1, b} .
$$

In contrast, our infinitesimal invariant $\delta\left[\mathcal{Z}_{d-3, a l g, b}\right]$ is the projection of

$$
\delta\left[\mathcal{Z}_{d-3, b}\right] \in \frac{\operatorname{Ker} \bar{\nabla}_{d-3, g-d+2, g-1, b} \subset \Omega_{B, b}^{d-3} \otimes H^{g-d+2, g-1}(J C)}{\operatorname{Im} \bar{\nabla}_{d-4, g-d+3, g-2}}
$$

modulo the subspace

$$
\Omega_{B, b} \wedge \operatorname{Ker} \bar{\nabla}_{d-4, g-d+2, g-1, b} \subset \operatorname{Ker} \bar{\nabla}_{d-3, g-d+2, g-1, b}
$$

which contains (4.33) by formula (2.7).

If instead of the family of plane curves, we consider the universal family of curves $\mathcal{C} \rightarrow B$ of genus $g$, and replace $d-3$ by any integer $s$ such that $g \geqslant 2 s+1$ (this is the range we are interested in by Conjecture 1.4), then at the general point $b \in B$, the subspace (4.33) can be in fact computed explicitly. The result is as follows.

Proposition 4.1. Let $C=\mathcal{C}_{b}$ be a general curve of genus $g \geqslant 2 s+1$. Then the kernel

$$
\operatorname{Ker}\left(\bar{\nabla}_{0, g-s-1, g-1, b}: H^{g-s-1, g-1}(J C) \rightarrow \Omega_{B, b} \otimes H^{g-s-2, g}\right)
$$

is equal to the image

$$
\operatorname{Im}\left([C] *: H^{g-s, g}(J C) \rightarrow H^{g-s-1, g-1}(J C)\right),
$$

where $[C] *$ is the Pontryagin product with the class of $C$ in $H^{g-1, g-1}(J C)$.

Notice that $\operatorname{Im}\left([C] *: H^{g-s, g}(J C) \rightarrow H^{g-s-1, g-1}(J C)\right)$ is also equal to the nonprimitive part of the cohomology

$$
\operatorname{Im}\left(\theta^{g-s-1}: H^{0, s}(J C) \rightarrow H^{g-s-1, g-1}(J C)\right) .
$$

Proof of Proposition 4.1. The proposition above is in fact a geometric translation of our result in [Voi05], namely the vanishing of the Koszul cohomology group $K_{k, 1}\left(C, K_{C}\right)$ for a general curve of genus $g$ in the range $g \leqslant 2 k+1$. This vanishing says that in this range, the kernel of the Koszul differential

$$
\operatorname{Ker}\left(\bigwedge^{k} H^{0}\left(C, K_{C}\right) \otimes H^{0}\left(C, K_{C}\right) \rightarrow \bigwedge_{k-1} H^{0}\left(C, K_{C}\right) \otimes H^{0}\left(C, 2 K_{C}\right)\right)
$$

is equal to the image of the natural map

$$
\bigwedge^{k+1} H^{0}\left(C, K_{C}\right) \rightarrow \bigwedge^{k} H^{0}\left(C, K_{C}\right) \otimes H^{0}\left(C, K_{C}\right)
$$

(also a Koszul differential). If we now identify $H^{0}\left(C, K_{C}\right)$ with $\bigwedge^{g-1} H^{0,1}(C)=\bigwedge^{g-1} H^{0,1}(C)$, the Koszul differential (4.36) identifies with the map $\bar{\nabla}_{0, k, g-1}, k=g-s-1$ of (4.35) because, as is well known, the multiplication map

$$
H^{0}\left(C, K_{C}\right) \otimes H^{0}\left(C, K_{C}\right) \rightarrow H^{0}\left(C, 2 K_{C}\right)
$$




\section{INFINITESIMAL INVARIANTS AND 1-CYCLES IN JACOBIANS}

seen in the form

$$
H^{0}\left(C, K_{C}\right) \rightarrow \operatorname{Hom}\left(H^{0}\left(C, K_{C}\right), H^{0}\left(C, 2 K_{C}\right)\right)=H^{0,1}(C) \otimes H^{0}\left(C, 2 K_{C}\right)
$$

identifies with the map $\bar{\nabla}_{0,1,0}$.

For $g \geqslant 2 s+1$, we have $g \leqslant 2(g-s-1)+1$. It thus follows from the above that the kernel of $\bar{\nabla}_{0, g-s-1, g-1}$ is equal to the image of $\bigwedge^{g-s} H^{0}\left(C, K_{C}\right)$. We finally observe that the natural map

$$
\bigwedge^{g-s} H^{0}\left(C, K_{C}\right) \rightarrow \bigwedge^{g-s-1} H^{0}\left(C, K_{C}\right) \otimes H^{0}\left(C, K_{C}\right)
$$

identifies with the map $[C] *: H^{g-s, g}(J C) \rightarrow H^{g-s-1, g-1}(J C)$ given by Pontryagin product with the class of $C$.

We refer to $[A N 10,8.1 .4$ for a more detailed version of this Hodge theoretic interpretation of the vanishing of canonical syzygies.

Proposition 4.1 shows that the Ikeda subspace (4.33) is too small to make the Ikeda infinitesimal invariant of the cycles $\mathcal{Z}_{s} \in \mathrm{CH}^{g-1}(\mathcal{J})$ for $g \geqslant 2 s+1$ invariant under relative translation of the cycle. Indeed, choose a degree 1 codimension $g$ cycle $\Gamma$ in $\mathcal{J}$ and consider the cycle

$$
\mathcal{Z}_{\Gamma}:=\mathcal{Z} * \Gamma,
$$

where $*$ is the relative Pontryagin product over $B$ for cycles in $\mathcal{J}$. Then

$$
\mathcal{Z}_{\Gamma}-\mathcal{Z}=\mathcal{Z} *\left(\Gamma-0_{B}\right)
$$

where $0_{B}$ is the zero section of $\mathcal{J}$. Since the 0 -cycles $\Gamma_{b}-0_{b} \in \mathrm{CH}_{0}\left(\mathcal{J}_{b}\right)$ are of degree 0 , they are algebraically equivalent to 0 , and so are the cycles $\mathcal{Z}_{\Gamma, b}-\mathcal{Z}_{b}$.

If we now introduce the relative Beauville decomposition

$$
\Gamma=\sum_{i} \Gamma_{i}, \Gamma_{0}=0_{B}
$$

of $\Gamma$, we find that

$$
\mathcal{Z}_{\Gamma, s}=\sum_{i \leqslant s} \mathcal{Z}_{s-i} * \Gamma_{i}
$$

In this sum, the first term is $\mathcal{Z}_{s}$ and the last term is $\mathcal{Z}_{0} * \Gamma_{s}$, and its restriction to $\mathcal{J}_{b}$ over the point $b \in B$ belongs by definition to the subgroup $Z_{0} F^{s} \mathrm{CH}_{g-1}\left(\mathcal{J}_{b}\right)$. So its infinitesimal invariant belongs by Ikeda's results to the Ikeda subspace (4.33). The other terms however have infinitesimal invariants which can be shown, using Proposition 4.1 and Ceresa's Theorem, not to belong to the Ikeda subspace (4.33) for $g \geqslant 2 s+1$ (while they, of course, belong to the subspace (4.34) by Proposition 2.4, since $\mathcal{Z}_{s-i} * \Gamma_{i}$ is algebraically equivalent to 0 for $i>0$ ).

\section{REFERENCES}

ACGH85 E. Arbarello, M. Cornalba, P. Griffiths and J. Harris, Geometry of algebraic curves, Vol. I, Grundlehren der Mathematischen Wissenschaften 267, Springer-Verlag, New York, 1985.

AN10 M. Aprodu and J. Nagel, Koszul cohomology and algebraic geometry, University Lecture Series, 52, American Mathematical Society, Providence, RI, 2010.

Bea86 A. Beauville, Sur l'anneau de Chow d'une variété abélienne, Math. Ann. 273 (1986), no. 4, 647-651. http://dx.doi.org/10.1007/BF01472135 


\section{Claire Voisin}

Bea04 A. Beauville, Algebraic cycles on Jacobian varieties, Compositio Math. 140 (2004), 683-688. http://dx.doi.org/10.1112/S0010437X03000733

Bei87 A. Beilinson, Height pairing between algebraic cycles in $K$-theory, arithmetic and geometry, (Moscow, 1984-1986), 1-25, Lecture Notes in Math., vol. 1289, Springer, Berlin, 1987. http: //dx.doi.org/10.1007/BFb0078364

Cer83 G. Ceresa, $C$ is not algebraically equivalent to $C^{-}$in its Jacobian, Ann. of Math. (2) 117 (1983), no. 2, 285-291. http://dx.doi.org/10.2307/2007078

CG80 J. Carlson and P. Griffiths, Infinitesimal variations of Hodge structure and the global Torelli theorem, in Géométrie algébrique, Angers 1979, ed. A. Beauville, 51-76, Sijthoff-Noordhoff, 1980.

CP95 A. Collino and G. P. Pirola, The Griffiths infinitesimal invariant for a curve in its Jacobian, Duke Math. J. 78 (1995), no. 1, 59-88.

CvG93 E. Colombo and B. van Geemen, Note on curves in a Jacobian, Compositio Math. 88 (1993), no. $3,333-353$.

Del68 P. Deligne, Théorème de Lefschetz et critères de dégénérescence de suites spectrales, Inst. Hautes Études Sci. Publ. Math. No. 35 (1968), 259-278.

Del71 P. Deligne, Théorie de Hodge II, Inst. Hautes Études Sci. Publ. Math. 40 (1971), 5-57.

Del91 P. Deligne, Décompositions dans la catégorie dérivée, in Motives (Seattle, WA, 1991), 115128, Proc. Sympos. Pure Math. 55, Part 1, Amer. Math. Soc., Providence, RI, 1994. http: //dx.doi.org/10.1090/pspum/055.1/1265526

DM91 C. Deninger and J. Murre, Motivic decomposition of abelian schemes and the Fourier transform, J. Reine Angew. Math. 422 (1991), 201-219.

EP94 H. Esnault and K. Paranjape, Remarks on absolute de Rham and absolute Hodge cycles, C. R. Acad. Sci. Paris Sér. I Math. 319 (1994), no. 1, 67-72.

Fak96 N. Fakhruddin, Algebraic cycles on generic Abelian varieties, Compositio Math. 100 (1996), 101-119.

vdGK07 G. van der Geer and A. Kouvidakis, Cycle relations on Jacobian varieties. With an appendix by Don Zagier, Compositio Math. 143 (2007), no. 4, 900-908.

Gri69 P. Griffiths, On the periods of certain rational integrals I, II, Ann. of Math. (2) 90 (1969), 460-495 and 496-541.

Gri83 P. Griffiths, Infinitesimal variations of Hodge structure, III, Determinantal varieties and the infinitesimal invariant of normal functions, Compositio Math. 50 (1983), no. 2-3, 267-324.

Her07 F. Herbaut, Algebraic cycles on the Jacobian of a curve with a linear system of given dimension, Compositio Math. 143 (2007), no. 4, 883-899.

Ike03 A. Ikeda, Algebraic cycles and infinitesimal invariants on Jacobian varieties, J. Algebraic Geom. 12 (2003), 573-603. http://dx.doi.org/10.1090/S1056-3911-03-00360-6

Mar08 G. Marini, Tautological cycles on Jacobian varieties, Collect. Math. 59 (2008), no. 2, 167-190. http://dx.doi.org/10.1007/BF03191366

Moo09 B. Moonen, Relations between tautological cycles on Jacobians, Comment. Math. Helv. 84 (2009), no. 3, 471-502. http://dx.doi.org/10.4171/CMH/170

Mur90 J. P. Murre, On the motive of an algebraic surface, J. Reine Angew. Math. 409 (1990), 190-204.

Nor93 M. Nori, Algebraic cycles and Hodge-theoretic connectivity, Invent. Math. 111 (1993), no. 2, 349-373. http://dx.doi.org/10.1007/BF01231292

Pol07 A. Polishchuk, Algebraic cycles on the relative symmetric powers and on the relative Jacobian of a family of curves, I, Selecta Math. (N.S.) 13 (2007), no. 3, 531-569. http://dx.doi.org/ $10.1007 / \mathrm{s} 00029-008-0049-9$

PR07 G. P. Pirola and C. Rizzi, Infinitesimal invariant and vector bundles, Nagoya Math. J. 186 (2007), 95-118. 


\section{INFINITESIMAL INVARIANTS AND 1-CYCLES IN JACOBIANS}

PS08 C. Peters and J. Steenbrink, Mixed Hodge structures, Ergebnisse der Mathematik und ihrer Grenzgebiete 3, 52, Springer-Verlag, Berlin, 2008.

Sai96 S. Saito, Motives and filtration on Chow groups, Invent. Math. 125 (1996), 149-196. http: //dx.doi.org/10.1007/s002220050072

Sai02 S. Saito, Higher normal functions and Griffiths groups, J. Algebraic Geom. 11 (2002), 161-201. http://dx.doi.org/10.1090/S1056-3911-01-00294-6

Voi88 C. Voisin, Une remarque sur l'invariant infinitésimal des fonctions normales, C. R. Acad. Sci. Paris 307, Série 1 (1988), 157-160.

Voi94a C. Voisin, Variations of Hodge structure and algebraic cycles, in Proceedings of the International Congress of Mathematicians, Vol. 1, 2, (Zürich, 1994), 706-715, Birkhäuser, Basel, 1995.

Voi94b C. Voisin, Variations de structure de Hodge et zéro-cycles sur les surfaces générales, Math. Ann. 299 (1994), 77-103. http://dx.doi.org/10.1007/BF01459773

Voi03 C. Voisin, Hodge Theory and complex algebraic geometry II, Cambridge University Press, 2003.

Voi04 C. Voisin, Remarks on filtrations on Chow groups and the Bloch conjecture, Ann. Mat. Pura Appl. (4) 183 (2004), no. 3, 421-438. http://dx.doi.org/10.1007/s10231-003-0092-2

Voi05 C. Voisin, Green's canonical syzygy conjecture for generic curves of odd genus, Compositio Math. 141 (2005), no. 5, 1163-1190. http://dx.doi.org/10.1112/S0010437X05001387

Voi14 C. Voisin, Chow rings, decomposition of the diagonal and the topology of families, Annals of Mathematics Studies 187, Princeton University Press, 2014.

Claire Voisin voisin@math.polytechnique.fr

Centre de mathématiques Laurent Schwartz, 91128 Palaiseau Cédex, France 